\title{
Focal adhesion kinase inhibitor TAE226 combined with Sorafenib slows down hepatocellular carcinoma by multiple epigenetics effects
}

Ilaria Romito

Bambino Gesu Pediatric Hospital: Ospedale Pediatrico Bambino Gesu

\section{Manuela Porru}

Regina Elena Institute Institute for Hospitalization and Care Scientific: Istituto Regina Elena

Maria Rita Braghini

Bambino Gesu Pediatric Hospital: Ospedale Pediatrico Bambino Gesu

Luca Pompili

Regina Elena Institute Institute for Hospitalization and Care Scientific: Istituto Regina Elena

Nadia Panera

Bambino Gesu Pediatric Hospital: Ospedale Pediatrico Bambino Gesu

\section{Annalisa Crudele}

Bambino Gesu Pediatric Hospital: Ospedale Pediatrico Bambino Gesu

\section{Daniela Gnani}

San Raffaele Hospital: IRCCS Ospedale San Raffaele

\section{Cristiano De Stefanis}

Bambino Gesu Pediatric Hospital: Ospedale Pediatrico Bambino Gesu

\section{Marco Scarsella}

Bambino Gesu Pediatric Hospital: Ospedale Pediatrico Bambino Gesu

Silvia Pomella

Bambino Gesu Pediatric Hospital: Ospedale Pediatrico Bambino Gesu

\section{Stefano Levi Mortera}

Bambino Gesu Pediatric Hospital: Ospedale Pediatrico Bambino Gesu

Emmanuel de Billy

Bambino Gesu Pediatric Hospital: Ospedale Pediatrico Bambino Gesu

\section{Libenzio Adrian Conti}

Bambino Gesu Pediatric Hospital: Ospedale Pediatrico Bambino Gesu

Valeria Marzano

Bambino Gesu Pediatric Hospital: Ospedale Pediatrico Bambino Gesu

\section{Lorenza Putignani}

Bambino Gesu Pediatric Hospital: Ospedale Pediatrico Bambino Gesu

\section{Manlio Vinciguerra}


University Hospital Brno: Fakultni Nemocnice Brno

\section{Clara Balsano}

University of L'Aquila Department of Experimental Medicine: Universita degli Studi dell'Aquila Dipartimento di Medicina Clinica Sanita Pubblica Scienze della Vita e dell'Ambiente

\section{Anna Pastore}

Bambino Gesu Pediatric Hospital: Ospedale Pediatrico Bambino Gesu

\section{Rossella Rota}

Bambino Gesu Pediatric Hospital: Ospedale Pediatrico Bambino Gesu

\section{Marco Tartaglia}

Bambino Gesu Pediatric Hospital: Ospedale Pediatrico Bambino Gesu

\section{Carlo Leonetti}

Regina Elena Institute Institute for Hospitalization and Care Scientific: Istituto Regina Elena

\section{Anna Alisi ( $\square$ anna.alisi@opbg.net )}

Bambino Gesu Pediatric Hospital: Ospedale Pediatrico Bambino Gesu

\section{Research Article}

Keywords: HCC, FAK, Sorafenib, epigenetics, therapy

Posted Date: September 16th, 2021

DOI: https://doi.org/10.21203/rs.3.rs-892488/v1

License: (c) (1) This work is licensed under a Creative Commons Attribution 4.0 International License. Read Full License

Version of Record: A version of this preprint was published at Journal of Experimental \& Clinical Cancer Research on November 16th, 2021. See the published version at https://doi.org/10.1186/s13046-02102154-8. 


\section{Abstract \\ Background}

Hepatocellular carcinoma (HCC) is one of the most common and lethal malignant tumours worldwide.

Sorafenib (SOR) is one of the most effective single-drug systemic therapy against advanced HCC, but the identification of novel combination regimens for a continued improvement in overall survival is a big challenge. Recent studies highlighted the crucial role of focal adhesion kinase (FAK) in HCC growth. The aim of this study was to investigate the antitumor effects of three different FAK inhibitors, alone or in combination with SOR, using in vitro and in vivo models of HCC.

\section{Methods}

The effect of PND1186, PF431396, TAE226 on cell viability was compared to SOR. Among them TAE226, emerging as the most effective FAKi, was then tested alone or in combination with SOR using 2D/3D human HCC cell line cultures and HCC xenograft murine models. The mechanisms of action were assessed by gene/protein expression and imaging approaches, combined with high-throughput methods.

\section{Results}

TAE226 emerged as the more effective FAKi to be combined with SOR against HCC. Combined TAE226 and SOR treatment reduced HCC growth both in vitro and in vivo by affecting tumour-promoting gene expression and inducing epigenetic changes via dysregulation of the nuclear interactome of FAK. We characterized a novel nuclear functional interaction between FAK and the NuRD complex. TAE226mediated FAK depletion and SOR-promoted MAPK down-modulation causing an increase of histone $\mathrm{H} 3$ lysine 27 acetylation, counteracting its trimethylation by decreasing the nuclear amount of HDAC1/2.

\section{Conclusions}

Altogether, our findings provide the first evidence that TAE226 combined with SOR efficiently reduce HCC growth in vitro and in vivo. Our data also highlight that deep analysis of FAK nuclear interactome may lead to the identification of new promising therapeutic approaches for HCC.

\section{Background}

Hepatocellular carcinoma (HCC) has been recognized as the fifth most common type of cancer, accounting for $70-85 \%$ of liver cancers, with an estimated 5 -year survival less than $20 \%$ [1]. HCC is notoriously resistant to systemic therapies, and often recurs after aggressive local therapies [2]. Currently, systemic therapies, have challenged the use of conventional therapies for HCC. Sorafenib (SOR), an orally available multi-kinase inhibitor, is one of the most effective single-drug systemic therapy and numerous 
clinical studies have shown that this drug provides good survival benefits in patients with advanced HCC [3-5].

However, SOR treatment causes several off-target and side effects, and response is transient due the occurrence of resistance, which is generally reported within 6 months of treatment. Several mechanisms have been implicated in the reduction of tumour cell sensitivity to SOR, including epigenetics, transport processes, regulated cell death, and the tumour microenvironment [6].

The partial success of SOR has generated enthusiasm in the development of new molecules to be used as first- or second-line agents for systemic treatment of HCC. Between 2017 and 2018, four drugs (Lenvatinib, Regorafenib, Cabozantinib, and Ramucirumab), were found to be effective and tolerated, and have been approved by the Food and Drug Administration (FDA) as first- or second-line therapeutic agents for HCC patients $[4,5,7,8]$. To minimize resistance that often occurs as a consequence of the multi-step nature of HCC pathogenesis, the use of multi-target and/or combined therapies are suggested as the optimal strategy. Indeed, Kudo et al. have recently reported the efficacy of combining transarterial chemoembolization (TACE) with SOR [9]. Therefore, considering that SOR currently remains the first-line agent for the treatment of unresectable HCC, it is imperative to identify new targetable molecules able to impair SOR resistance by investigating signaling pathways that are dysregulated in HCC onset and progression.

Recently, studies highlighted that focal adhesion kinase (FAK), often activated by autophosphorylation at Tyr-397 (pTyr397FAK) and over-expressed in liver cancer, could be one potential druggable target to fight HCC [10-12]. Accordingly, Gnani et al. [13] showed that FAK depletion reduces HCC cell growth by affecting cancer-promoting genes including the pro-oncogene EZH2. This study unveiled a previously unappreciated FAK/Enhancer of zeste homolog 2 (EZH2) crosstalk in HCC cells, thus identifying a targetable network paving the way for new anticancer therapies. Besides that, it has been suggested that the use of FAK inhibitors (FAKi) in combination with other FDA approved therapies could be a promising therapeutic option for HCC [14]. In particular, PND1186 effectively inhibits Dasatinib induced pTyr397FAK expression, and synergizes with Dasatinib to block HCC cell growth in vitro by decreasing proliferation and inducing apoptosis [14]. Interestingly, Azzariti et al. [15] demonstrated that SOR resistance could be caused by the crosstalk between the tissue microenvironment and HCC through the hepatic stellate cells secreting Ln-332, a major ligand for a3 31 integrin that leads to recovery the ubiquitinated FAK by SOR.

A number of FAKi are commercially available and some of them have been tested in clinical trials in other tumour types, such as glioblastoma and lung cancer $[16,17]$. To date, no studies have evaluated the performance of these drugs in reducing $\mathrm{HCC}$ cell growth either alone or in combination with SOR.

Here, we investigated the effects on HCC cells viability of three different FAKi: PND1186, PF431396, and TAE226. We deeply analysed the antitumor effects of TAE226, which emerged as the most effective FAKi, alone or in combination with SOR either in vitro or in vivo HCC models. 
We found that TAE226 combined with SOR efficiently reduces HCC growth by exerting multiple epigenetic effects strongly associated to FAK nuclear interactome.

\section{Methods}

\section{Cell lines}

The human HepG2 cells were purchased from ATCC (Manassas, Virginia, USA) that provided certificated authentication, and previously certified Huh7 cells. All cell lines were grown in Dulbecco's modified Eagle's medium supplemented with $10 \%$ FBS, $1 \%$ L-glutamine, and antibiotics (1\% penicillin/streptomycin) at $37^{\circ} \mathrm{C}$ in $5 \% \mathrm{CO}_{2}$ in a $95 \%$ humidified atmosphere. All cells used for this study resulted negative to the presence of Mycoplasma spp. after testing by Venor GeM Advance Mycoplasma Detection Kit (Minerva Biolabs, Berlin, Deutschland).

To follow in vivo tumour growth, HepG2 cells were stably transfected with an inducible plasmid encoding firefly luciferase and selected with G418 to produce HepG2-Luc cells as already described [13].

\section{Treatments}

SOR, PND1186, and PF431396 (Selleck Chemicals, Houston, TX, USA) and TAE226 (kindly provided by Novartis Pharm) were dissolved in dimethyl sulfoxide and stored at $-80^{\circ} \mathrm{C}$ until time of use. HCC cells were exposed to different concentration of drugs for different times as detailed in the results.

Cells were lentivirally silenced for FAK as already described [13].

\section{Cell viability assay}

Cell viability was evaluated by using a colorimetric assay (XTT based) for the non-radioactive quantification of cell proliferation and viability (Roche Molecular Biochemicals, Indianapolis, Indiana, USA), according to the manufacturer's protocol. The absorbance of the water-soluble formazan formed was measured at $490 \mathrm{~nm}$ using the ELISA Benchmark Plus microplate spectrophotometer (Bio-Rad Laboratories, Hercules, California, USA). The viability of the treated cells was expressed as the percentage of killed cells versus untreated cells. The Combination Index $(\mathrm{Cl})$ was calculated with the Chou-Talalay method using CalcuSyn v.2.0 software in order to calculate the synergism, additivity or antagonism of the different drug combinations [18].

\section{Cell proliferation assay}

Cells were plated in a 96 microplate well. Next, 5-bromo-2'deoxyuridine (BrdU) assay was performed by using the Dissociation-Enhanced Lanthanide Fluorescent Immunoassay (DELFIA) Cell Proliferation Kit following the manufacturer instructions (Perkin Elmer, Waltham, Massachusetts, USA). The fluorescence, which is proportional to DNA-synthesis, was measured by time-resolved fluorometer 2100 EnvisionTM Multilabel Reader (Perkin Elmer).

\section{Cell cycle and apoptosis analysis}


Cell cycle phase distribution was analysed by flow cytometry using propidium iodide (PI) staining (SigmaAldrich, St. Louis, Missouri, USA). HCC cells were collected by trypsinization, washed with PBS, then fixed in a cold solution of methanol/acetone (4:1). Cells were first incubated with RNase $A$ at $37^{\circ} \mathrm{C}$ then stained with a solution containing $100 \mu \mathrm{g} / \mathrm{ml} \mathrm{PI}$, at $37^{\circ} \mathrm{C}$ for 20 minutes. Stained nuclei were analysed for DNA-PI fluorescence using a Becton Dickinson FACSCanto II flow cytometer (Becton-Dickinson, Milan, Italy). The proportions of cells in G0/G1, S phase, and G2/M phases of the cell cycle by DiVa Software, version 6.3 (Becton-Dickinson).

Apoptosis was assessed by FITC Annexin V Apoptosis Detection Kit (Becton-Dickinson). Briefly, cells were washed in PBS and re-suspended in Annexin Binding Buffer (10mmol/L HEPES pH 7.4, $140 \mathrm{mmol} / \mathrm{L} \mathrm{NaCl}$, and $2.5 \mathrm{mmol} / \mathrm{L} \mathrm{CaCl}$ ). Cells were then stained with $0.5 \mu$ Annexin V-FITC and $5 \mu \mathrm{l} \mathrm{PI} \mathrm{(Becton-Dickinson)}$ for 15 minutes before analysing. Acquisition and analysis were carried out on a Becton Dickinson FACSCanto II flow cytometer, using DiVa Software, version 6.3.

\section{Western Blotting (WB)}

Cells were collected and total protein extraction was performed by RIPA lysis buffer (Cell Signaling Technology, Danvers, Massachusetts, USA) containing 1X protease and phosphatase inhibitors cocktail. The homogenates were then centrifuged at $13000 \mathrm{rpm}$ at $4^{\circ} \mathrm{C}$ for 10 minutes and the resulting supernatant was taken as protein sample. Whole cell extracts were quantified using the BCA Protein Assay (Thermo Fisher Scientific Inc., Waltham, Massachusetts, USA). Samples were then diluted in the sample buffer (200 mM Tris- $\mathrm{HCl}$ ( $\mathrm{pH}$ 6.8), 40\% glycerol, 20\% $\beta$-mercaptoethanol, 4\% sodium dodecyl sulphate, and bromophenol blue) and resolved in SDS-PAGE, then transferred and immobilized onto nitrocellulose membranes (Amersham, Little Chalfont, UK). The membranes were blocked using 5\% nonfat dry milk for 30 minutes and incubated with the appropriate primary and secondary antibodies. Protein expression was quantified by densitometry analysis using Image J v3.91 software. The used antibodies are listed in Table $\mathrm{S} 1$.

\section{Spheroid generation and image acquisition}

200 cells/well of HepG2 and 400 cells/well of Huh7 were seeded in $100 \mu \mathrm{l}$ of complete growth medium on 96 Ultra-Low Attachment (Corning Life Sciences, Amsterdam, Netherlands) well plates to obtain, 96 hours after cell seeding, tumour spheroids (TS) of $250 \mu \mathrm{m}$ in diameter. Forty-eight hours after treatments, diameters and area were calculated and TS were stained using PI (1 mg/ml final concentration). Image capture and analysis were performed using Celigo imaging cytometer (Nexcelom Biosciences LLC, Lawrence, Kansas, USA).

\section{Anchorage-independent soft agar colony formation assay}

HCC cells were treated as expected from the experiment and then mixed with $0.3 \%$ agarose and were plated over a $0.6 \%$ agarose layer. The medium was renewed twice weekly. After $21-30$ days, the cells were stained with $0.05 \%$ crystal violet solution and counted manually. The data are represented as number of colonies per well. 


\section{Animals}

Animal procedures were in compliance with the national and international directives (D.L. 4 March 2014, no. 26; directive 2010/63/EU of the European Parliament and of the council; Guide for the Care and Use of Laboratory Animals, United States National Research Council, 2011) and approved by the Italian Ministry of Health (authorization n. 213/2019-PR, released date 03/13/2019).

Six to eight week-old CD1-nude or NOD/SCID male mice were used (Charles River laboratories, Calco, Milan, Italy) to establish xenograft model of HCC as already reported [13]. Briefly, HepG2-Luc were intramuscularly (heterotopic) injected in nude mice at $5 \times 10^{6} \mathrm{cells} / \mathrm{mice}$ for pilot study or injected into the liver (orthotopic) of NOD/SCID mice at $10^{6} \mathrm{cells} / \mathrm{mice}$. Treatments started when a well-established tumour (4-5 mm) was evident in mice (day 19 after tumour cells injection) as measured by a caliper in heterotopic tumours, while mice bearing intrahepatic tumours were imaged using the IVIS imaging system 200 series (Perkin Elmer).

In the heterotopic pilot study, 8 CD1-nude mice for each group of treatment were used to identify the adequate concentration of TAE226 for in vivo setting of the drug. Therefore, at day 19 , the animals were divided in three groups and treated orally by gavage as follows: Group 1A: Vehicle; Group 2A: TAE226 at $25 \mathrm{mg} / \mathrm{kg}$ for two weeks; Group 3A: TAE226 at $50 \mathrm{mg} / \mathrm{kg}$ for two weeks.

In order to evaluate the effect of combination therapy, at day 18 the orthotopically injected NOD/SCID mice $(n=24)$ were divided in four groups and treated as follows: Group 1B: Vehicle; Group 2B: TAE226 at $25 \mathrm{mg} / \mathrm{kg}$ orally for five consecutive days repeated for 2 weeks; Group 3B: SOR at $30 \mathrm{mg} / \mathrm{kg}$ orally for five consecutive days; Group 4B: TAE226 at $25 \mathrm{mg} / \mathrm{kg}$ for five consecutive days repeated for 2 weeks followed by SOR at $30 \mathrm{mg} / \mathrm{Kg}$ for five consecutive days.

Mice were analysed by imaging at different times before tumour cells injection and during the treatment. At day 35 from the start of treatments the animals were sacrificed. Imaging data were acquired at different timepoints (days from treatment 0, 14, 21, 28 and 35) and analysed using the Living Image Software version 4.3 (Perkin Elmer).

\section{Immunofluorescence}

Immunofluorescence was performed on $2 \mu$ m-thick sections obtained from formalin-fixed tissue embedded in paraffin. Antigen retrieval was performed with EDTA (pH 8) (Dako, Glostrup, Denmark). The sections were incubated overnight with specific primary antibodies at $4^{\circ} \mathrm{C}$ (Table S1).

The primary antibodies were revealed with the secondary antibodies purchased by Alexa Fluor (Thermo Fisher Scientific Inc.). The confocal microscopy imaging was performed on Olympus Fluoview FV1000 confocal microscope equipped with FV10-ASW version 2.0 software, using 20X and 40X objective. Fluorochromes unmixing was performed by acquisition of automated-sequential collection of multi- 
channel images, in order to reduce spectral crosstalk between channels. The sections were incubated overnight with specific primary antibodies at $4^{\circ} \mathrm{C}$.

\section{Gene expression and pathways enrichment}

A pre-designed TaqMan OpenArray Human Cancer Panel (Thermo Fisher Scientific Inc.) was used to assess the effect of FAK inhibition plus SOR in HepG2 and Huh7 cells on a signature panel of 624 welldefined genes validated for the characterization of cancers, plus 24 endogenous controls. cDNAs were loaded onto the Open Array platform and run as recommended by the manufacturer on the QuantStudio 12K Flex Real-Time PCR system (Thermo Fisher Scientific Inc.). Relative gene expression values were calculated as relative quantity (RQ) by using Open Source expression suite provided by Life Technologies (Thermo Fisher Scientific Inc.). RQ minimum and maximum values were calculated with a confidence level of $95 \%$, using Benjamini-Hochberg false discovery rate (FDR) to adjust $p$ values. Maximum allowed Ct included in calculations were 35.

Pathway analysis was conducted by querying Reactome annotations using the R/Bioconductor library reactome.db $[19,20]$. For Reactome analysis, only pathways with a FDR lower than 0.05 and $p<0.05$ were considered.

\section{Detection of histone H3 Lysine 27 trimethylation}

HepG2 and Huh7 cells were cultured and then treated with the combined therapy or with the single inhibitors. At the end of the treatment, AlphaLISA assay was conducted using the Tri-Methyl-Histone H3 Lysine 27 (H3K27me3) Cellular Detection Kit (Perkin Elmer) following the manufacturer's instructions. Detection was performed with an EnVision Multilabel Reader (Perkin Elmer) using the AlphaScreen standard settings as previously described [21].

\section{Cell imaging}

Cells were cultured in a 96 well collagen coated plate (Perkin Elmer) and treated with combined therapy TAE226 plus SOR. Next, cells were fixed with a solution of methanol/acetone (3:1) at $-20^{\circ} \mathrm{C}$ for 20 minutes. After two brief washes with PBS, cells were blocked with PBS/BSA 1\% at room temperature (RT) for 30 minutes and then incubated with pTyr397FAK antibody (Table S1) diluted in PBS/BSA $1 \%$ for 2 hours at RT. Then, cells were washed twice with PBS and incubated with 1:500 Alexa Fluor 488 goat antirabbit IgG secondary antibody (Thermo Fisher Scientific Inc.) in PBS/BSA 1\% for 30 minutes at RT. Next, cells were washed with PBS and then incubated with 1:15000 Hoechst in PBS/BSA 1\% for 5 minutes at RT for nuclear staining.

Cell imaging was performed by Operetta CLS in confocal mode (Perkin Elmer) and the 40X water immersion objective. Image segmentation and analysis were performed with the Harmony software 4.8 (Perkin Elmer) for the determination of the mean intensity by wells of the fluorescence signal related to cytoplasmic and nuclear pTyr397FAK.

\section{Nuclear protein extraction}


For extraction of nuclear protein, cells were washed with PBS, trypsinized, pelleted by centrifugation at $1200 \mathrm{rpm}$ for 5 minutes and then resuspended in the cell lysis buffer [10 mM HEPES pH 7.5, $10 \mathrm{mM} \mathrm{KCl}$, $0.1 \mathrm{mM}$ EDTA, $1 \mathrm{mM}$ dithiothreitol (DTT), 0.5\% Nonidet-40 and $0.5 \mathrm{mM}$ PMSF along with the $1 \mathrm{x}$ protease and phosphatase inhibitor cocktail (Thermo Fisher Scientific Inc.)] and kept in ice for 15-20 minutes with intermittent mixing. Then, tubes have been vortexed to disrupt cell membranes and centrifuged at 12000 $\mathrm{g}$ at $4^{\circ} \mathrm{C}$ for 10 minutes.

The pelleted nuclei were washed with the cell lysis buffer, resuspended in the nuclear extraction buffer [20 mM HEPES pH 7.5, $400 \mathrm{mM} \mathrm{NaCl}, 1$ mM EDTA, 1 mM DTT, 1 mM PMSF with 1x protease and phosphatase inhibitor cocktail] and then incubated in ice for 30 minutes. Nuclear extract was collected by centrifugation at $12000 \mathrm{~g}$ for 15 minutes at $4^{\circ} \mathrm{C}$. Protein concentration was estimated using BCA Protein Assay. The extract is either immediately used or stored at $-80^{\circ} \mathrm{C}$ until further use.

\section{Immunoprecipitation}

Immunoprecipitation samples (500 $\mathrm{\mu g}$ nuclei) were pre-cleared with protein A/G PLUS-Agarose beads (Santa Cruz Biotechnology, Dallas, Texas, USA) for 2 hours at $4^{\circ} \mathrm{C}$ under end-to-end rotation. At the end of the incubation, the samples were washed with TBS, centrifuged for $30 \mathrm{~s}$ at $5000 \mathrm{~g}$ at RT, and supernatant was recovered. Next, $20 \mu \mathrm{l}$ of conjugates between beads and primary antibody (Table S1) were added to supernatant and incubated for 2 hours at $4^{\circ} \mathrm{C}$ under end-to-end rotation. Negative controls (Mock) were performed with protein A/G PLUS-Agarose beads alone (without adding antibody to the extract). At the end of the incubation, the beads were centrifuged for $30 \mathrm{~s}$ at $5000 \mathrm{~g}$ at RT, and supernatant was discarded. Then, the immunoprecipitates were washed three times by adding $200 \mu$ of TBS, were eluted in sample buffer and resolved by $12 \%$ SDS-PAGE.

\section{Proteomic analysis}

Immunoprecipitates were separated by mono-dimensional SDS-PAGE and resolved proteins were visualized with QC Colloidal Coomassie stain (Bio-Rad Laboratories). Gel lanes were excised into 10 bands and in-gel digested [22]. Extracted peptides were subjected to nanoLiquid ChromatographyElectroSpray lonization-tandem Mass Spectrometry (nLC-ESI-MS/MS) analysis performed on an UltiMate3000 RSLCnano System directly coupled to the Orbitrap Fusion Tribrid mass spectrometer (Thermo Fisher Scientific Inc.). The peptide mixtures were first trapped and desalted onto a $\mu$-precolumn C18 PepMap100 (5 $\mu$ m particle size, $100 \AA ̊$ pore size, $300 \mu \mathrm{m}$ i.d. x 5mm length, Thermo Fisher Scientific Inc.) for 3 minutes at $10 \mu \mathrm{L} /$ minutes with an aqueous solution of $2 \%$ acetonitrile (ACN) and $0.1 \%$ trifluoroacetic acid, and then separated by reverse-phase chromatography performed on an EASY-Spray PepMap RSLC C18 column ( $2 \mu \mathrm{m}$ particle size, $100 \AA$ A pore size, $75 \mu \mathrm{m}$ i.d. x $50 \mathrm{~cm}$ length, Thermo Fisher Scientific Inc.) at a flow rate of $250 \mathrm{~nL} /$ minutes, at a temperature of $35^{\circ} \mathrm{C}$, by a one-step linear gradient starting from $98 \%$ eluent $A(0.1 \%$ formic acid, $F A$, in water) to $25 \%$ eluent $B(99.9 \% A C N, 0.1 \% F A)$ in 60 minutes, and a total LC-run of 96 minutes. Orbitrap detection was used for precursor (MS1) ions measurements at resolving powers of $120 \mathrm{~K}$ (at $200 \mathrm{~m} / \mathrm{z}$ ), whereas fragments (MS2, MS/MS) ions were recorded by lon Trap at rapid scan rate. Data dependent MS/MS analysis was performed in top speed 
mode with a 3 s cycle-time, during which most abundant multiple-charged (2+ $-7+)$ precursor ions detected within the range of $250-1,500 \mathrm{~m} / \mathrm{z}$ were selected for activation in order of abundance. The signal intensity threshold for MS2 was $5 \times 10^{3}$. Quadrupole isolation with a $1.6 \mathrm{~m} / \mathrm{z}$ isolation window was used, and dynamic exclusion was enabled for 1 minute. High-energy collisional dissociation was performed using $30 \%$ normalized collision energy. Automatic gain control targets were $4.0 \times 10^{5}$ for MS and $2 \times 10^{3}$ for MS2, with 50 and 300 ms maximum injection times, respectively. The option "Injection lons for All Available Parallelizable Time" was set.

Proteins were identified with the SequestHT algorithm embedded in the Proteome Discoverer software (version 1.4, Thermo Fisher Scientific Inc.) by merging the 10 raw data files for each lane in a single analysis and interrogating the Homo sapiens UniProtKB reference proteome (ID: UP000005640, release: 2020_03, 20,621 sequence entries). The search parameters were set to use a tolerance of $10 \mathrm{ppm}$ and 0.6 Da for precursor ions and product ions respectively, allowing 1 missed cleavage. Carbamidomethylation of cysteine was set as fixed modification while oxidation of methionine, phosphorylation of serine, tyrosine and threonine, and protein $\mathrm{N}$-terminal acetylation were chosen as variable modifications.

A FDR threshold of 0.01 , using Percolator algorithm for PSM validation.

Results were filtered in all experiments considering only those identified proteins with at least two unique peptides and a SumPEP score value $\geq 50$. Keratines were also filtered out from the resulting protein list.

\section{Analysis of protein-protein interaction (PPI) networks}

The STRING (Search Tool for Recurring Instances of Neighbouring Genes) database [23] was used for characterization of nuclear interactors of FAK. PPI networks between nuclear interactors of FAK were identified by typing proteins names in the software along with the selection of the species under investigation ( $H$. sapiens). In order to exclude most of the false positive interactions as possible, we used 0.7 (high level of confidence) as the total STRING protein interaction confidence scores and six number of clusters for PPI filtering. Gene Ontology (GO) enrichment analysis was carried out by using STRING online tools.

\section{Detection of histone H3 Lysine 27 acetylation}

AlphaLISA assay was conducted similarly to the H3K27me3 analysis by using the AlphaLISA AcetylatedHistone H3 Lysine 27 (H3K27ac) Cellular Detection Kit (Perkin Elmer).

\section{Statistics}

Multivariate Student's t-test or one-way ANOVA were applied and values of $p<0.05$ were considered statistically significant. GraphPad Prism 5.0 was used for statistical analysis.

\section{Results}




\section{Effects of FAKi on cell viability, cell proliferation and FAK autophosphorylation in HCC cells}

We first looked at the sensitivity of HepG2 and Huh7 HCC cell lines to three different commercially available FAKi (PND1186, PF431396, TAE226), and to SOR. We performed a dose response assay to determine the half-maximal inhibitory concentration values (IC50) following 48 hours of treatment. As shown in Fig. 1A-1D, all compounds reduced cell viability evaluated as percentage of killed cells. In particular, the following values of IC50 were determined: PND1886 7.1 $\mu \mathrm{M}$ for HepG2 cells and $10 \mu \mathrm{M}$ for Huh7 cells (Fig. 1A); PF431396 10.3 $\mu \mathrm{M}$ for HepG2 cells and 5 $\mu \mathrm{M}$ for Huh7 cells (Fig. 1B); TAE226 11.7 $\mu \mathrm{M}$ for HepG2 cells and $4.1 \mu \mathrm{M}$ for Huh7 cells (Fig. 1C); SOR 11.3 $\mu \mathrm{M}$ for HepG2 cells and $5.7 \mu \mathrm{M}$ for Huh7 cells (Fig. 1D). In both cell lines, we also evaluated the cell proliferation rate after 48 hours of treatment with the different drugs administered at their IC50 values, compared with not-treated (NT) cells (Fig. 1E). From this analysis, SOR and TAE226 resulted as the most efficient in reducing cell proliferation in HepG2 cells, while SOR proved to be the most effective treatment, followed by PF431396 and TAE226 in Huh7 cells.

As shown in Fig. 1F and Fig. S1, TAE226 was the most effective drug in causing a reduction of pTyr397FAK in HepG2, and the second one for efficacy in Huh7 cells. Based on these findings, we selected TAE226 as the FAKi to be studied alone and in combination with SOR on HCC models.

\section{TAE226 in combination with SOR efficiently reduces HCC cell viability and proliferation}

In order to assess the timing for evaluating TAE226 and SOR effects we treated HepG2 and Huh7 cells with IC50 of each inhibitor up to 5 days of treatment. A statistically significant decrease of cell viability occurred after just 2 days of treatment with both drugs, and was further enhanced after 5 days of treatment (Fig. S2).

Afterwards, to identify the combination of the two drugs (TAE226 and SOR) with major effects on cell viability, HCC cells were cultured in the medium for: i) 5 days (NT); ii) 1 day, then treated for 2 days with TAE226 alone, and finally exposed to SOR alone until day 5 (TAE226 >SOR); iii) 1 day, then treated for 2 days with SOR, and finally exposed to TAE226 alone until day 5 (SOR > TAE226); iv) 1 day, then treated for 4 days contemporaneously with TAE226 and SOR (TAE226 + SOR); as schematic represented in Fig. 2A. As shown in Fig. 2B and 2C, the combination therapy TAE226 > SOR was the most efficient into reducing cell viability. The same combination therapy was also the most effective in decreasing DNA-synthesis (Fig. 2D) compared to other combinations and to NT cells.

TAE226 plus SOR combination treatment impairs FAK phosphorylation at Tyr397 and enhances in vitro antitumor effect.

Next, we evaluated the downstream effects of the selected drug-combination (TAE226 > SOR) compared with those of TAE226 and SOR alone and with NT cells (Fig. 3A). 
Immunoelectrophoretic profile and densitometric analysis revealed that HCC cells treated with combination therapy TAE226 > SOR exhibited a strong reduction of pTyr397FAK protein levels (Fig. 3B and 3C). Moreover, as previously reported by Fukami et al. [24], TAE226 alone or in combination with SOR confirmed to be able to down-regulate also the pTyr1135 form of the insulin growth factor 1 receptor $\beta$ (pTyr1135IGF-1R), a well-known tyrosine kinase receptor crucial for tumour transformation and malignant cell survival (Fig. S3A and S3B).

Our results also demonstrated that the treatment with TAE226 as single agent or in combination with SOR (TAE226 > SOR) caused a significant reduction in the percentage of HCC cells in G0/G1 and S phase of the cell cycle, and an accumulation in G2/M phase compared to NT cells (Fig. 3D). On the contrary, SOR treatment, used as single agent, increased the percentage of HCC cells in S phase to the detriment of those in G0/G1 phase (Fig. 3D). Data on cell death highlighted that TAE226 alone, and even more in combination with SOR (TAE226 > SOR), induced an increase of the percentage of cells in early and late apoptosis, while SOR caused cell death (Fig. 3E).

To better mimic the physiological response to treatments, their inhibitory effects after 48 hours was also evaluated on 3D multicellular TS obtained from HepG2 and Huh7 cells. From phase contrast images (brightfield) (Fig. 3F) and measurements (Fig. S3C and S3D) emerged that TAE226 and SOR treatments, alone or in combination, caused a reduction of TS area and diameter. Moreover, TAE226 was competent both alone and even more in combination with SOR to increase TS cell death as showed by PI staining (Fig. 3F).

The antitumor effect of treatments was also demonstrated by their inhibition of 28-days colonies formation when compared to the colonies formed by NT cells (Fig. S3E).

\section{TAE226 enhances the antitumor effect of SOR in in vivo HCC models}

Based on the collected promising in vitro results, we performed experiments to assess the antitumor efficacy of TAE226 > SOR combination in vivo. First, we evaluated the safe/effective dosage of TAE226 on intramuscular tumour bearing mice treated with $25 \mathrm{mg} / \mathrm{kg}$ and $50 \mathrm{mg} / \mathrm{kg}$ of TAE226, starting from day 19 after the injection of HepG2-Luc cells. The effect of the selected doses on the reduction of tumour growth was almost the same after 12 days of treatment without toxicity effects on the animals (Fig. S4A), as shown by the similar levels of alanine aminotransferase (ALT) in treated mice compared to those of untreated animals (Fig. S4B). Therefore, we selected $25 \mathrm{mg} / \mathrm{kg}$ as TAE226 concentration to perform experiments on orthotopic HepG2 HCC model and treated mice by the scheduling reported in Fig. 4A to evaluate the in vivo therapeutic efficacy of TAE226 and SOR. The antitumor efficacy was evaluated by using the IVIS imaging system. As reported in Fig. 4B, we observed that the combination treatment TAE226 > SOR showed antitumor efficacy against orthotopic tumours. In fact, tumour growth was significantly inhibited at $21-28$ days after initiation of treatment of about than $50 \%$ by TAE226 or SOR when used as single treatment, after than a regrowth of tumour was observed. Of note, combination therapy was able to significantly affect tumour growth for a very long period being already observed at 
day 21 with a reduction of tumour weight of about $90 \%$ and the effect was maintained until day 28 with a reduction of about $70 \%$. Interestingly, the efficacy of TAE226 to increase the potency of SOR in this advanced model of human HCC was confirmed when we applied the response evaluation criteria in solid tumours [25]. In fact, stabilization of the disease for about three weeks was observed in 4/6 mice and partial response in 1/6 mice treated with the combination (Table S2). Accordingly, as shown by immunofluorescence analysis performed on tumours at day 35 (Fig. 4C), the expression levels of proliferating cell nuclear antigen (PCNA) strongly decreased only in combination group compared to single treatments and untreated animals. Finally, as expected, the levels of pTyr397FAK expression tended to be reduced both by TAE226 and SOR alone, but were more strongly down-regulated in TAE226 $>$ SOR (Fig. 4D).

\section{Combination therapy TAE226> SOR affects the expression of cancer-related genes}

Since we previously demonstrated that FAK silencing impacts on gene transcription [13], we wanted to evaluate if this effect was causally related to FAK catalytic activity. Thus, we analysed the effect of FAK pharmacological inhibition on the expression of a panel of cancer-related genes in HepG2 and Huh7 cells after TAE226 > SOR treatment compared to NT cells. We analysed the expression of 648 genes using a commercially available cancer open array by Real-Time PCR platform. TAE226 $>$ SOR combined treatment induced an up-regulation of 161 genes in HepG2 cells and 59 genes in Huh7 cells (Fig. S5A), whilst it caused a down-regulation of 176 genes in HepG2 cells and 66 genes in Huh7 cells (Fig. S5B). Venn diagrams (Fig. 5A) showed that a set of 13 and 27 potential direct/indirect specific targets were respectively upregulated and down-modulated in both cell lines compared to NT cells. Next, we performed Reactome enrichment analysis, considering separately the common up-regulated genes and those downregulated (Table S3). As shown in Fig. 5B, among the enriched terms, we found up-regulated pathways related to biological oxidations and acetylation, and down-regulated pathways associated to signal transduction, gene transcription and extracellular matrix (ECM) degradation. Genes belonging to the immune system were both up- and down-regulated.

Combination therapy TAE226 > SOR affects the tri-methylation of lysine 27 on histone H3 and nuclear amount of pTyr397FAK

We previously observed that FAK-dependent deregulation of gene expression results in a reduced $E Z H 2$ expression and tri-methylation of lysine 27 on histone $\mathrm{H} 3$, with consequent effects on gene transcription [13]. Therefore, we explored the effect of TAE226 inhibitor alone or in combination with SOR on the same pathway. As shown in Fig. 6A, AlphaLISA assay demonstrated that H3K27me3 was significantly downregulated by TAE226 or SOR treatment in both HCC cell lines. This effect was more pronounced in cells cultured with the TAE226 > SOR combined treatment. Accordingly, EZH2 protein expression levels were significantly decreased by all treatments (Fig. 6B and 6C). These findings support the hypothesis that pTyr397FAK is deeply involved in the observed effects on H3K27me3, however we still needed to explore cellular compartments in which the combination treatment plays its antitumor effects in HCC cells. 
Representative images showed that TAE226 > SOR reduced nuclear and focal adhesion localization of pTyr397FAK in HCC cell lines (Fig. 7A). The content of pTyr397FAK into the nuclei and at focal adhesions was quantified by using Operetta, an automated imaging system (Fig. 7B and 7C). Similarly, in xenograft mice models of HCC, the treatment with TAE226 > SOR caused a down-regulation of the amount of pTyr397FAK, at levels of cytoplasm as well as at focal contacts and nuclei (Fig. 7D).

\section{Combination therapy TAE226 > SOR reduces pTyr397FAK nuclear interaction with specific epigenetic regulators}

We previously suggested that the effect of FAK on H3K27me3 could be mediated by direct and/or indirect functional interactions between FAK and EZH2 [13]; the mechanism by which pTyr397FAK may influence H3 tri-methylation, however, remained to be explored. In order to identify the nuclear interactors of FAK that could participate to the epigenetic control of gene expression, we performed proteomics on nuclear immunoprecipitates of FAK obtained from HepG2 and Huh7 cells (Fig. S6A). The identified proteins were next used for further analysis (Fig. S6B). We identified 116 putative nuclear FAK binding partners (Table S4), including the well-known $\beta$-catenin functional interactor of FAK [26]. To better understand the nuclear interactome of FAK and the crosstalk of these proteins with known pathways, we constructed the interaction network containing nodes corresponding to FAK interacting proteins and further integrated the information on their associated pathways by using STRING database [23], and six protein-protein interaction (PPI) networks were generated (Fig. 8A). GO annotations (Table S5) suggest that FAK interactors are mainly enriched in molecular functions of proteins involved in protein stability (proteasome), RNA splicing (spliceosome), and DNA binding (epigenetic regulators). Among these proteins, some components of nucleosome remodelling and deacetylase (NuRD) complex, a chromatinremodelling complex having a histone deacetylase activity, emerged as engaging putative nuclear interactors of FAK. Indeed, as demonstrated by immunoprecipitation experiments, nuclear FAK interacted with the deacetylase NuRD-proteins HDAC1 and HDAC2, and FAK silencing abolished this interaction (Fig. 8B). Moreover, in HCC cells treated with TAE226 > SOR the decrease of pTyr397FAK levels was associated with a reduction of $\beta$-catenin, HDAC1 and HDAC2 (Fig. $8 \mathrm{C}$ and Fig. S7). The HDACs nuclear impairment after TAE226 > SOR treatment in both HCC cells was coupled with the consequent upregulation of $\mathrm{H} 3 \mathrm{~K} 27 \mathrm{ac}$ (Fig. 8D).

\section{Discussion}

In this study, we provided a clear and comprehensive preclinical evaluation of a FAK inhibitor, TAE226, studied in HCC cells in order to explore its potential use as first-line therapy in combination with SOR.

We found that TAE226 alone, but especially in combination with SOR, efficiently reduced growth and increased apoptosis of 2D and 3D HCC in vitro models. Moreover, TAE226 slowed down tumour growth of human HCC xenografts in vivo, and cooperated with SOR enhancing the antitumor activity. Furthermore, the combination treatment did not induced more side effects respect to the monotherapy. 
FAK is a highly conserved $125 \mathrm{kDa}$ non-receptor tyrosine kinase, mainly localized to cellular focal contacts, playing a master role in adhesion-dependent cell motility, survival and proliferation, in response to integrin and growth factor receptor signalling via its kinase-dependent and scaffold functions $[27,28]$. During the last decade, the role of FAK in tumours and the utility of its inhibitors as potential therapeutic agents have been extensively investigated [29]. Even though FAK was identified as an independent risk factor for HCC, with its overexpression predicting poor prognosis in $\mathrm{HCC}$ patients, currently there is little evidence of the role of FAK activation, and studies on the effect of clinically translatable FAK inhibitors are still in embryo in this tumour $[30,31]$. We previously demonstrated that FAK silencing may reduce in vitro and in vivo HCC growth by affecting cancer-promoting genes, including the pro-oncogenic EZH2 [13]. Moreover, it has been reported that FAK may play a key role in the control of liver cancer stem cells proliferation, thus its inhibition and functional interaction with $\beta$-catenin has recently been identified as a potential strategy to overcome SOR-related resistance associated to this cell subpopulation [31, 32]. All these findings suggest that the network between FAK, epigenetic modifications and $\beta$-catenin might be a useful therapeutic target to treat HCC in presence of SOR resistance.

Here, we demonstrated that TAE226 strongly reduced HCC growth and appear to be more effective in combination with SOR, let thinking that the combined therapy could overpass SOR resistance in the treatment of HCC patients. The higher efficiency of TAE226 in reducing HCC growth respect to other FAK inhibitors could be linked to its ability of targeting also IGF-1R phosphorylation [24].

We found that combination therapy TAE226 plus SOR reduced HCC growth both in vitro and in vivo and affected the expression of tumour-promoting genes and multifunctional epigenetics changes via deregulation of the nuclear interactome of FAK. In particular, combination therapy induced changes in genes involved in the immune system, signal transduction, ECM degradation, and intriguingly emerged the effect on acetylation regulators. The epigenetic effect of FAK depletion in HCC anticipated by our previous study has been confirmed by the strong reduction of EZH2 protein expression and H3K27me3 after TAE226 plus SOR combined treatment [13].

The multiple epigenetic effects of FAK inhibition suggested that this protein might exert some of these functions in the nuclear compartment. Accordingly, several lines of evidence have demonstrated that, into the nucleus, activated FAK may control different transcription factors leading to changes in gene expression [33]. Our work demonstrate that the nuclear localization of the Tyr397 phosphorylated form of FAK in HCC cells was reverted by the combined treatment TAE226 plus SOR in both in vitro and in vivo models. Moreover, to define the nuclear interactome of FAK in HCC, we performed a proteomic analysis. The results of proteomic study confirmed the evidence of multiple functional but also physical interactions of FAK with $\beta$-catenin, spliceosome, proteasome, and epigenetic regulators $[13,34-36]$. Noteworthy, in our study the physical interaction of FAK with the NuRD complex emerged as a great novelty of the nuclear interactome of FAK. The NuRD complex consists of different subunits, including histone deacetylase HDAC1/HDAC2, the histone demethylase KDM1A, MTA1/MTA2/MTA3, GATAD2A/GATAD2B, RBBP4/RBBP7, MBD2/MBD3, and the ATP-dependent chromatin remodelling helicase CHD3/CHD4 [37]. Although NuRD complex has been linked to many aspects of oncogenesis, the 
expression and regulation of its subunits are not completely understood in HCC [38-40]. Our results highlighted that either FAK depletion by silencing or combined treatment TAE226 plus SOR decreased the nuclear amount of HDAC1/2, reducing their activity by an increase of H3K27Ac. It is conceivable that the increased acetylation of histone $\mathrm{H} 3$ lysine 27 observed under the treatment with TAE226 plus SOR may act as a recovery mechanism of the expression of tumour suppressor genes that are maintained inactive by histone $\mathrm{H} 3$ lysine 27 methylation in HCC. However, this aspect requires further investigation.

\section{Conclusions}

In conclusion, as reported in Fig. 8E, we found that TAE226 inhibitor of FAK combined with SOR slows down HCC growth by multifunctional epigenetics effects, which mainly include the reduction of FAK nuclear amount and its detrimental activity on histone $\mathrm{H} 3$ acetylation. Taken together, our findings provide the evidence that the modulation of FAK nuclear interactome may lead to new promising therapeutics for HCC [35].

\section{Abbreviations}

HCC: hepatocellular carcinoma; SOR: sorafenib; FAK: focal adhesion kinase; FDA: food and drug administration; TACE: transarterial chemoembolization; pTyr397FAK: phosphorylated at Tyr-397 form of FAK; EZH2: enhancer of zeste homolog 2; FAKi: FAK inhibitors; Cl: combination index; DELFIA: dissociation-enhanced lanthanide fluorescent immunoassay; PI: propidium iodide; WB: western blotting; TS: tumour spheroids; H3K27me3: tri-methyl-histone H3 lysine 27; RT: room temperature; PPI: proteinprotein interaction; GO: gene ontology; H3K27ac: acetylated-histone H3 lysine 27; NT: not-treated; pTyr1135IGF-1R: phosphorylated at Tyr1135 form of the insulin growth factor 1 receptor $\beta$; ALT: alanine aminotransferase; PCNA: proliferating cell nuclear antigen; ECM: extracellular matrix; NuRD: nucleosome remodelling and deacetylase; IP: immunoprecipitated.

\section{Declarations}

Ethics approval and consent to participate: Animal procedures were in compliance with the national and international directives (D.L. 4 March 2014, no. 26; directive 2010/63/EU of the European Parliament and of the council; Guide for the Care and Use of Laboratory Animals, United States National Research Council, 2011) and approved by the Italian Ministry of Health (authorization n. 213/2019-PR, released date 03/13/2019).

Consent for publication: Not applicable

Availability of data and materials: The datasets used and/or analysed during the current study are available from the corresponding author on reasonable request.

Competing interests: The authors declare that they have no competing interests. 
Funding: The study was supported by Associazione Italiana per la Ricerca sul Cancro (AIRC, MFAG12936), Fondazione Bambino Gesù-ONLUS, and Italian Ministry of Health (Ricerca Corrente 2019) to AA and (Ricerca Corrente 2020) to LP.

Authors' contributions: IR, MP, CL and AA were responsible of conception and design of the study. IR, MP, MRB, LP, NP, AC, DG, CDS, MS, SP, SLM, EDB, ALC and VM executed and analysed the experiments. IR, MP, MRB and $A A$ interpreted the data and wrote the manuscript. MV, CB, LP, AP, RR, MT and CL edited and critical revised the manuscript. All authors read and approved the final manuscript.

Acknowledgements: The authors would like to thank Dr. Shinji Hatakeyama from Novartis Pharma for providing the TAE226 drug.

\section{References}

1. Bertuccio P, Turati F, Carioli G, Rodriguez T, La Vecchia C, Malvezzi M, et al. Global trends and predictions in hepatocellular carcinoma mortality. J Hepatol. 2017. doi:10.1016/j.jhep.2017.03.011.

2. Dhanasekaran R, Nault JC, Roberts LR, Zucman-Rossi J. Genomic Medicine and Implications for Hepatocellular Carcinoma Prevention and Therapy. Gastroenterology. 2019. doi:10.1053/j.gastro.2018.11.001.

3. Cheng AL, Kang YK, Chen Z, Tsao CJ, Qin S, Kim JS, et al. Efficacy and safety of sorafenib in patients in the Asia-Pacific region with advanced hepatocellular carcinoma: a phase III randomised, doubleblind, placebo-controlled trial. Lancet Oncol. 2009. doi:10.1016/S1470-2045(08)70285-7.

4. Zhou J, Sun $\mathrm{H}$, Wang Z, Cong W, Wang J, Zeng M, et al. Guidelines for the Diagnosis and Treatment of Hepatocellular Carcinoma (2019 Edition). Liver Cancer. 2020. doi:10.1159/000509424.

5. Llovet JM, Kelley RK, Villanueva A, Singal AG, Pikarsky E, Roayaie S, et al. Hepatocellular carcinoma. Nat Rev Dis Primers. 2021. doi:10.1038/nrdp.2016.18.

6. Tang W, Chen Z, Zhang W, Cheng Y, Zhang B, Wu F, et al. The mechanisms of sorafenib resistance in hepatocellular carcinoma: theoretical basis and therapeutic aspects. Signal Transduct Target Ther. 2020. doi:10.1038/s41392-020-0187-x.

7. Llovet JM, Montal R, Sia D, Finn RS. Molecular therapies and precision medicine for hepatocellular carcinoma. Nat Rev Clin Oncol. 2018. doi:10.1038/s41571-018-0073-4.

8. Niu M, Yi M, Li N, Wu K, Wu K. Advances of Targeted Therapy for Hepatocellular Carcinoma. Front Oncol. 2021. doi:10.3389/fonc.2021.719896.

9. Kudo M, Ueshima K, Ikeda M, Torimura T, Tanabe N, Aikata H, et al. Randomised, multicentre prospective trial of transarterial chemoembolisation (TACE) plus sorafenib as compared with TACE alone in patients with hepatocellular carcinoma: TACTICS trial. Gut. 2020. doi:10.1136/gutjnl-2019318934.

10. Fujii T, Koshikawa K, Nomoto S, Okochi O, Kaneko T, Inoue S, et al. Focal adhesion kinase is overexpressed in hepatocellular carcinoma and can be served as an independent prognostic factor. J 
Hepatol. 2004. doi:10.1016/j.jhep.2004.03.029.

11. Gillory LA, Stewart JE, Megison ML, Nabers HC, Mroczek-Musulman E, Beierle EA. FAK Inhibition Decreases Hepatoblastoma Survival Both In Vitro and In Vivo. Transl Oncol. 2013. doi:10.1593/tlo. 12505.

12. Chen JS, Huang $X H$, Wang Q, Chen XL, Fu XH, Tan $H X$, et al. FAK is involved in invasion and metastasis of hepatocellular carcinoma. Clin Exp Metastasis. 2010. doi:10.1007/s10585-010-93063.

13. Gnani D, Romito I, Artuso S, Chierici M, De Stefanis C, Panera N, et al. Focal adhesion kinase depletion reduces human hepatocellular carcinoma growth by repressing enhancer of zeste homolog 2. Cell Death Differ. 2017. doi:10.1038/cdd.2017.34.

14. Tanjoni I, Walsh C, Uryu S, Tomar A, Nam JO, Mielgo A, et al. PND-1186 FAK inhibitor selectively promotes tumor cell apoptosis in three-dimensional environments. Cancer Biol Ther. 2010. doi:10.4161/cbt.9.10.11434.

15. Azzariti A, Mancarella S, Porcelli L, Quatrale AE, Caligiuri A, Lupo L, et al. Hepatic stellate cells induce hepatocellular carcinoma cell resistance to sorafenib through the laminin-332/a3 integrin axis recovery of focal adhesion kinase ubiquitination. Hepatology. 2016. doi:10.1002/hep.28835.

16. Brown NF, Williams M, Arkenau HT, Fleming RA, Tolson J, Yan L, et al. A study of the focal adhesion kinase inhibitor GSK2256098 in patients with recurrent glioblastoma with evaluation of tumor penetration of [11C]GSK2256098. Neuro Oncol. 2018. doi:10.1093/neuonc/noy078.

17. Gerber DE, Camidge DR, Morgensztern D, Cetnar J, Kelly RJ, Ramalingam SS, et al. Phase 2 study of the focal adhesion kinase inhibitor defactinib (VS-6063) in previously treated advanced KRAS mutant non-small cell lung cancer. Lung Cancer. 2020. doi:10.1016/j.lungcan.2019.10.033.

18. Chou TC. Drug combination studies and their synergy quantification using the Chou-Talalay method. Cancer Res. 2010. doi:10.1158/0008-5472.CAN-09-1947.

19. Milacic M, Haw R, Rothfels K, Wu G, Croft D, Hermjakob H, et al. Annotating cancer variants and anticancer therapeutics in reactome. Cancers (Basel). 2012. doi:10.3390/cancers4041180.

20. Fabregat A, Sidiropoulos K, Garapati P, Gillespie M, Hausmann K, Haw R, et al. The Reactome pathway Knowledgebase. Nucleic Acids Res. 2016. doi:10.1093/nar/gkv1351.

21. Qian J, Lu L, Wu J, Ma H. Development of multiple cell-based assays for the detection of histone H3 Lys27 trimethylation (H3K27me3). Assay Drug Dev Technol. 2013. doi:10.1089/adt.2013.515.

22. Shevchenko A, Tomas H, Havlis J, Olsen JV, Mann M. In-gel digestion for mass spectrometric characterization of proteins and proteomes. Nat Protoc. 2006. doi:10.1038/nprot.2006.468.

23. STRING v.11 Database. https://string-db.org. Accessed 28 April 2021.

24. Fukami S, Tomioka D, Murakami Y, Honda T, Hatakeyama S. Pharmacological profiling of a dual FAK/IGF-1R kinase inhibitor TAE226 in cellular and in vivo tumor models. BMC Res Notes. 2019. doi:10.1186/s13104-019-4389-7. 
25. Gao H, Korn JM, Ferretti S, Monahan JE, Wang Y, Singh M, et al. High-throughput screening using patient-derived tumor xenografts to predict clinical trial drug response. Nat Med. 2015. doi:10.1038/nm.3954.

26. Shang N, Wang H, Bank T, Perera A, Joyce C, Kuffel G, et al. Focal Adhesion Kinase and $\beta$-Catenin Cooperate to Induce Hepatocellular Carcinoma. Hepatology. 2019. doi:10.1002/hep.30707.

27. Dawson JC, Serrels A, Stupack DG, Schlaepfer DD, Frame MC. Targeting FAK in anticancer combination therapies. Nat Rev Cancer. 2021. doi:10.1038/s41568-021-00340-6.

28. Brami-Cherrier K, Gervasi N, Arsenieva D, Walkiewicz K, Boutterin MC, Ortega A, et al. FAK dimerization controls its kinase-dependent functions at focal adhesions. EMBO J. 2014. doi:10.1002/embj.201386399.

29. Panera N, Crudele A, Romito I, Gnani D, Alisi A. Focal Adhesion Kinase: Insight into Molecular Roles and Functions in Hepatocellular Carcinoma. Int J Mol Sci. 2017. doi:10.3390/ijms18010099.

30. Fujii T, Koshikawa K, Nomoto S, Okochi O, Kaneko T, Inoue S, et al. Focal adhesion kinase is overexpressed in hepatocellular carcinoma and can be served as an independent prognostic factor. $\mathrm{J}$ Hepatol. 2004. doi:10.1016/j.jhep.2004.03.029.

31. Francalanci P, Giovannoni I, De Stefanis C, Romito I, Grimaldi C, Castellano A, et al. Focal Adhesion Kinase (FAK) Over-Expression and Prognostic Implication in Pediatric Hepatocellular Carcinoma. Int J Mol Sci. 2020. doi:10.3390/ijms21165795.

32. Fan Z, Duan J, Wang L, Xiao S, Li L, Yan X, et al. PTK2 promotes cancer stem cell traits in hepatocellular carcinoma by activating Wnt/ß-catenin signaling. Cancer Lett. 2019. doi:10.1016/j.canlet.2019.02.040.

33. Rivas Serna IM, Romito I, Maugeri A, Lo Re O, Giallongo S, Mazzoccoli G, et al. A Lipidomic Signature Complements Stemness Features Acquisition in Liver Cancer Cells. Int J Mol Sci. 2020. doi:10.3390/ijms21228452.

34. Tancioni I, Miller NL, Uryu S, Lawson C, Jean C, Chen XL, et al. FAK activity protects nucleostemin in facilitating breast cancer spheroid and tumor growth. Breast Cancer Res. 2015. doi:10.1186/s13058015-0551-x.

35. Mousson A, Sick E, Carl P, Dujardin D, De Mey J, Rondé P. Targeting Focal Adhesion Kinase Using Inhibitors of Protein-Protein Interactions. Cancers (Basel). 2018. doi:10.3390/cancers10090278.

36. Zhou J, Yi Q, Tang L. The roles of nuclear focal adhesion kinase (FAK) on Cancer: a focused review. J Exp Clin Cancer Res. 2019. doi:10.1186/s13046-019-1265-1.

37. Allen HF, Wade PA, Kutateladze TG. The NuRD architecture. Cell Mol Life Sci. 2013. doi:10.1007/s00018-012-1256-2.

38. Lee YH, Seo D, Choi KJ, Andersen JB, Won MA, Kitade M, et al. Antitumor effects in hepatocarcinoma of isoform-selective inhibition of HDAC2. Cancer Res. 2014. doi:10.1158/0008-5472.CAN-13-3531.

39. Zhou H, Cai Y, Liu D, Li M, Sha Y, Zhang W, et al. Pharmacological or transcriptional inhibition of both HDAC1 and 2 leads to cell cycle blockage and apoptosis via p21Waf1/Cip1 and p19INK4d upregulation in hepatocellular carcinoma. Cell Prolif. 2018. doi:10.1111/cpr.12447. 
40. Shao S, Cao H, Wang Z, Zhou D, Wu C, Wang S, et al. CHD4/NuRD complex regulates complement gene expression and correlates with CD8 T cell infiltration in human hepatocellular carcinoma. Clin Epigenetics. 2020. doi:10.1186/s13148-020-00827-3.

\section{Figures}

A

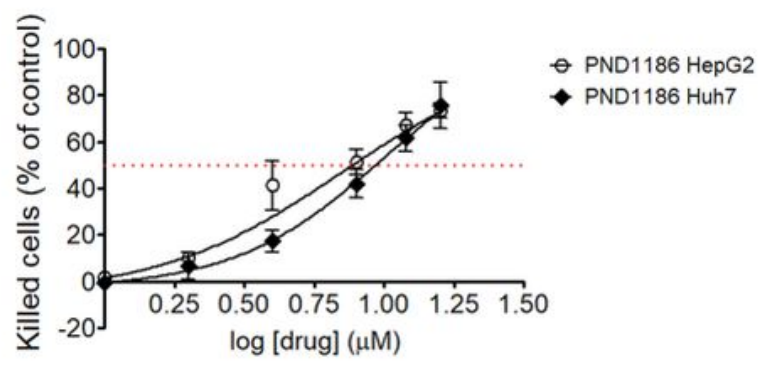

C

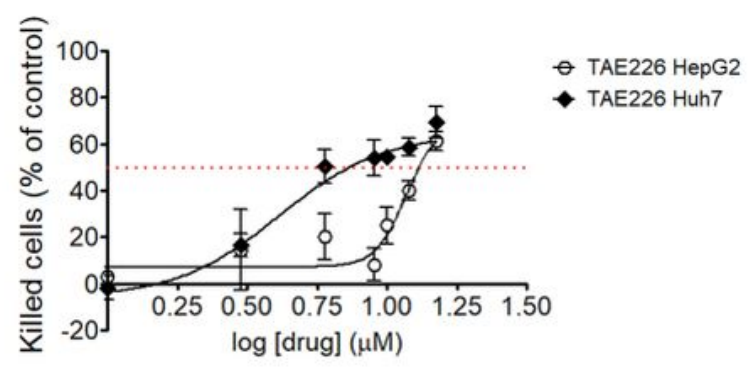

E

HepG2

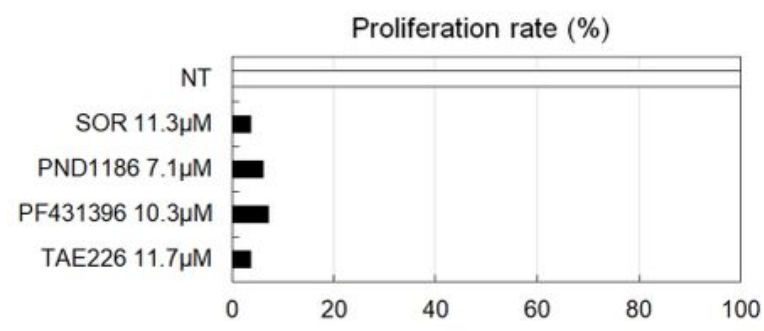

$\mathbf{F}$

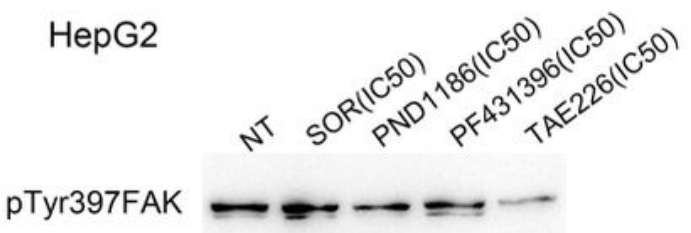

B

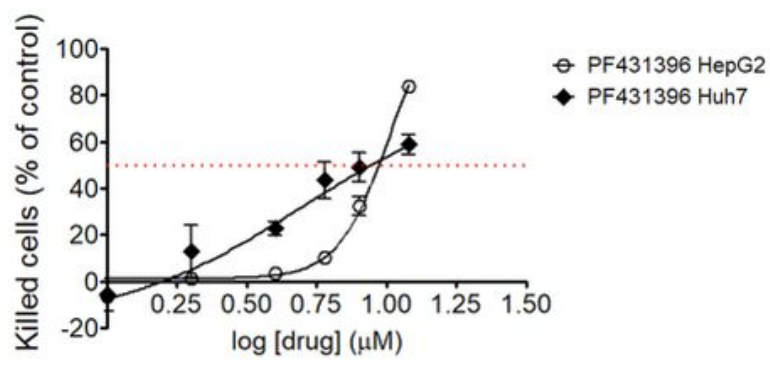

D

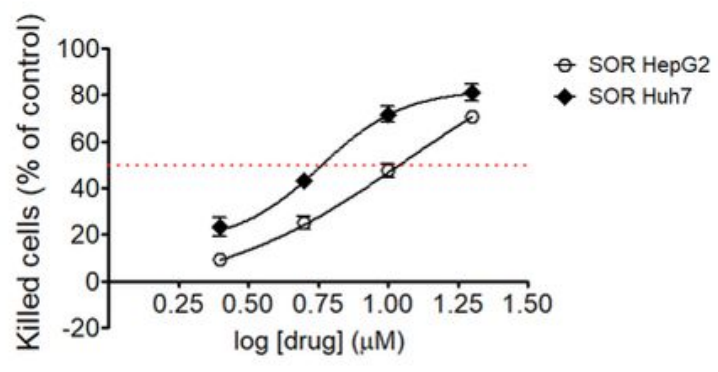

Huh7
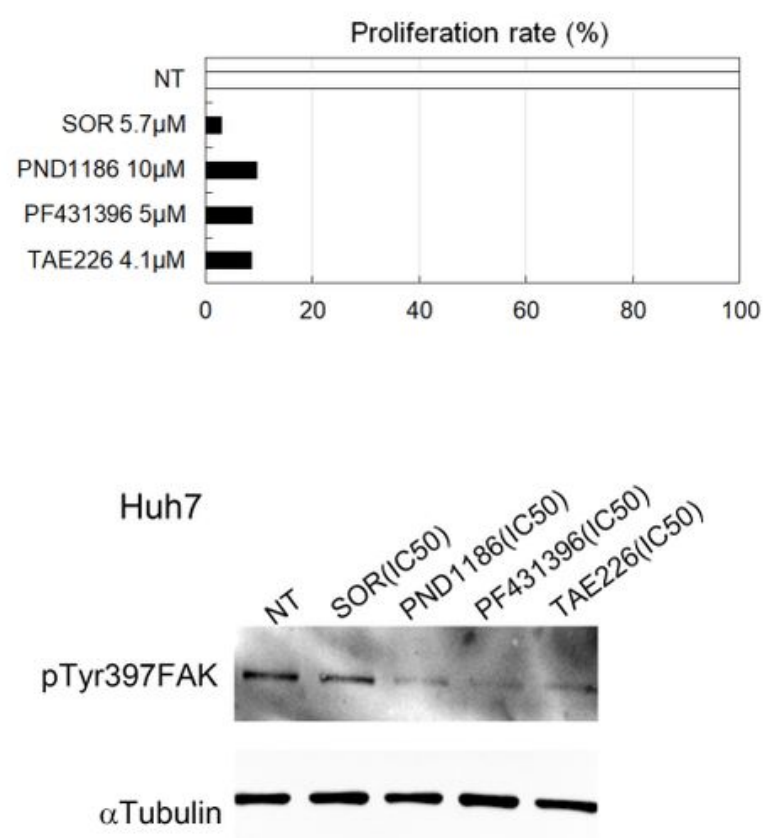
Effect of drugs on cell viability, cell proliferation and FAK phosphorylation in HCC cells. Dose-response curves and IC50 fit lane for PND1886 (A), PF431396 (B), TAE226 (C), and SOR (D) in HepG2 and Huh7 cells. Values are measured by XTT assay after 48 hours from treatment and expressed as percentage of killed cells respect to NT \pm Standard Deviation (SD) of three independent experiments. (E) Proliferation rate in HCC cells after treatment with IC50 values in HepG2 and Huh7 cells. Values are expressed as percentage respect to NT cells of the mean of at least three independent experiments. (F) Representative immunoblot by WB of pTyr397FAK expression after 48 hours from treatment with the different drugs, in HepG2 and Huh7 cells. 
A

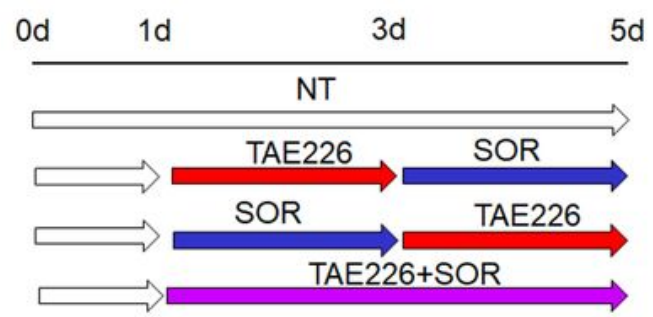

B

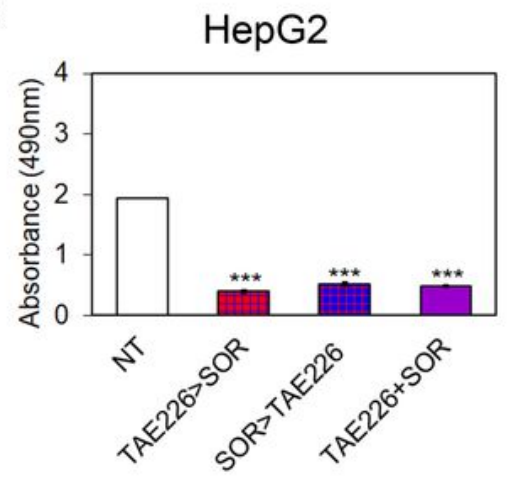

C

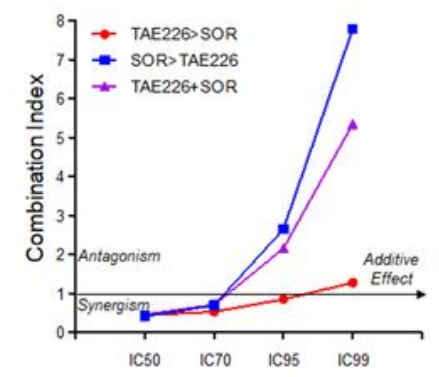

D

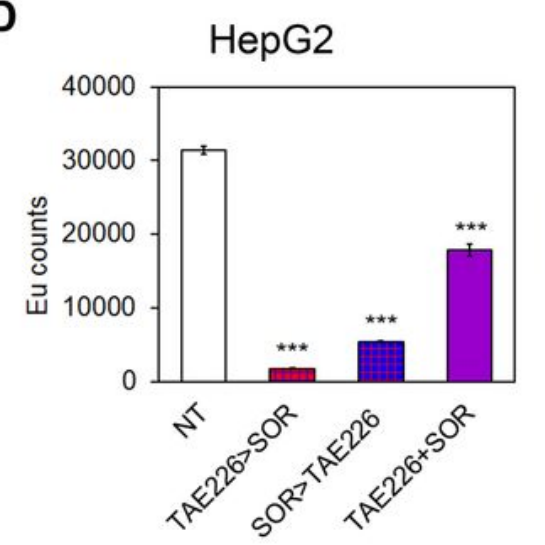

Huh7

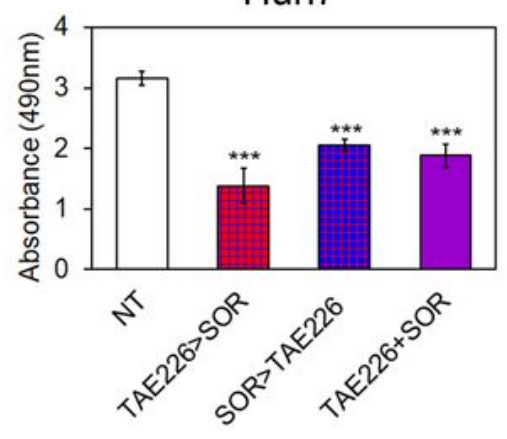

Huh7

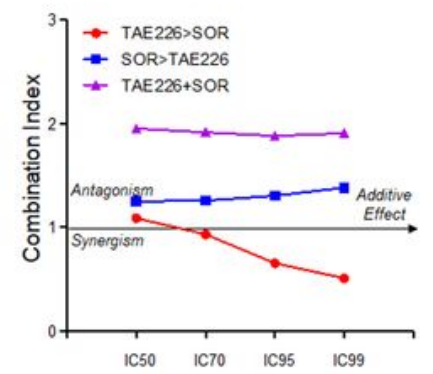

Huh7

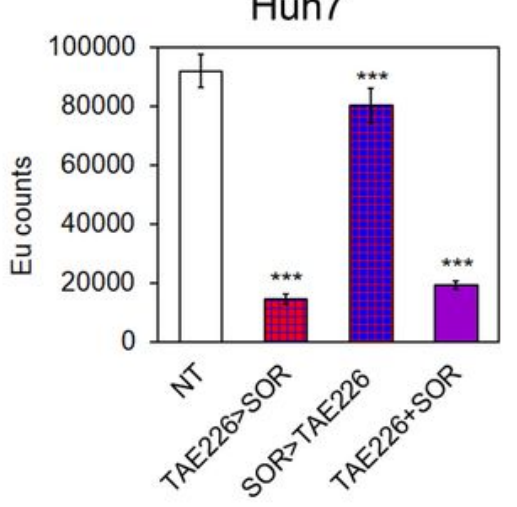

\section{Figure 2}

Viability and proliferation in HCC cells after treatment with different combination of TAE226 and SOR. (A) Scheme of the experimental design. (B) Cell viability, measured by XTT assay, in HepG2 and Huh7 cells treated vs. NT. Values are the mean OD \pm SD of three independent experiments repeated at least in triplicate. Data were analyzed by 2-tailed Student's t test. ***p $<0.001$. (C) Combination Index (CI) was calculated by the Chou-Talalay method. Data plotted are $\mathrm{Cl}$ at 50\%, 70\%, 95\% and 99\% fraction killed. (D) 
DNA-synthesis expressed as Europium (Eu) counts of BrdU incorporation in HepG2 and Huh7 cells treated vs. NT. Values are the mean Eu counts \pm SD of three independent experiments repeated at least in triplicate. Data were analyzed by 2 -tailed Student's $t$ test. ${ }^{* \star *} p<0.001$.

A

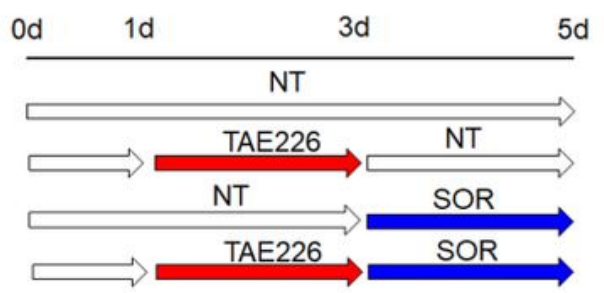

D

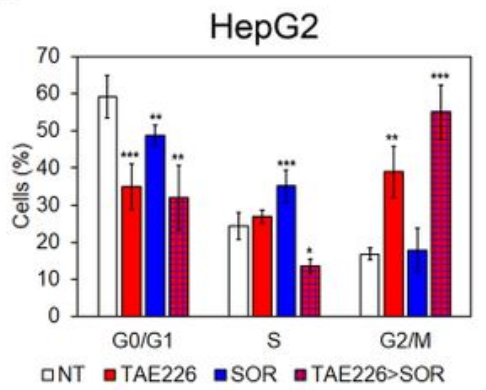

$E$
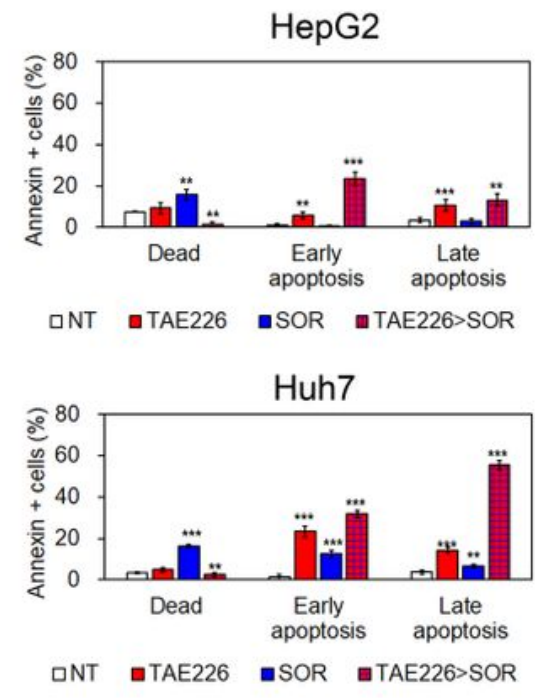

B

HepG2
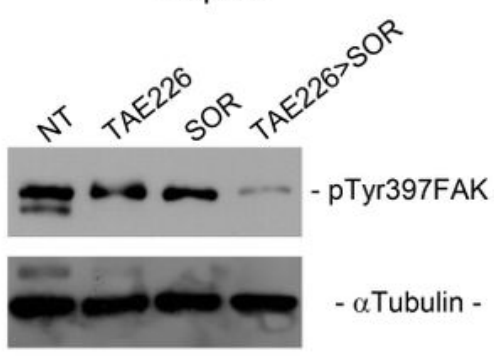

$-\alpha$ Tubulin -
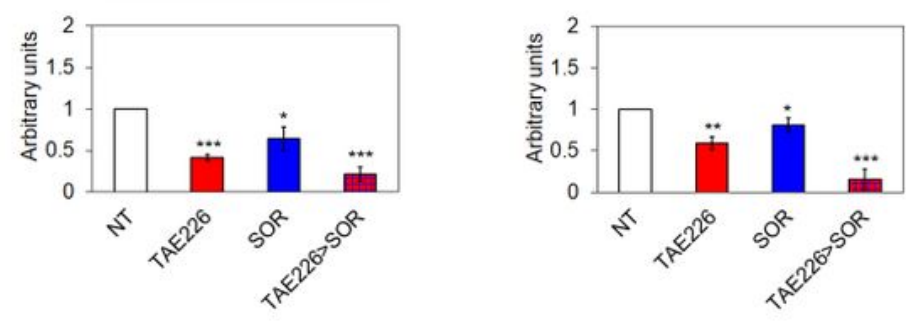

$\mathbf{F}$

Huh7

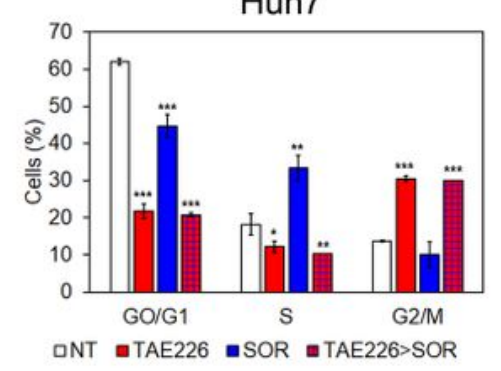

C

Huh7

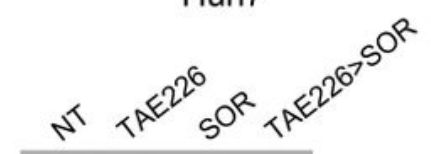


of pTyr397FAK expression after 48 hours from treatment with the different drugs, in HepG2 (B) and Huh7 cells $(C)$. Values are the mean arbitrary units \pm SD of at least three independent experiments. Data were analyzed by 2-tailed Student's $t$ test. ${ }^{*} p<0.05 ;{ }^{* \star} p<0.01$; ${ }^{* \star} p<0.001$ vs. NT. (D) Percentage of HCC cells in $\mathrm{G} 0 / \mathrm{G} 1, \mathrm{~S}$ and $\mathrm{G} 2 / \mathrm{M}$ phase of the cell cycle by PI staining and flow cytometry analysis. Data are expressed as mean $\pm S D$ of at least three independent experiments and was analyzed by 2-tailed Student's $t$ test. ${ }^{\star} p<0.05 ;{ }^{* \star} p<0.01 ;{ }^{* \star} p<0.001$ vs. NT. (E) Percentage of HCC cells dead or in early and late apoptosis measured by Annexin $V$ staining and flow cytometry. Data are expressed as mean \pm SD of at least three independent experiments and was analyzed by 2-tailed Student's $t$ test. ${ }^{\star \star} p<0.01$; ${ }^{\star \star \star} \mathrm{p}<$ 0.001 vs. NT. (F) Representative brightfield and fluorescent images (Hoechst and PI) of multicellular TS from HepG2 and Huh7 cells NT and after treatments. 
A

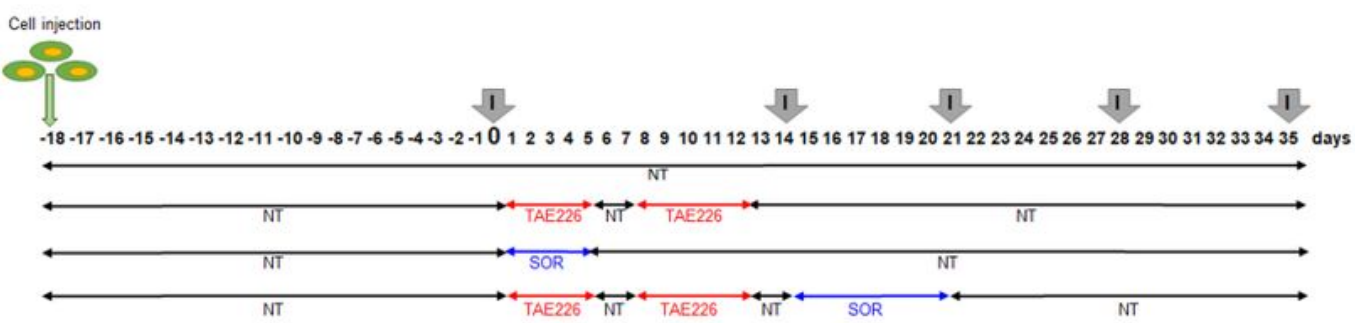

B

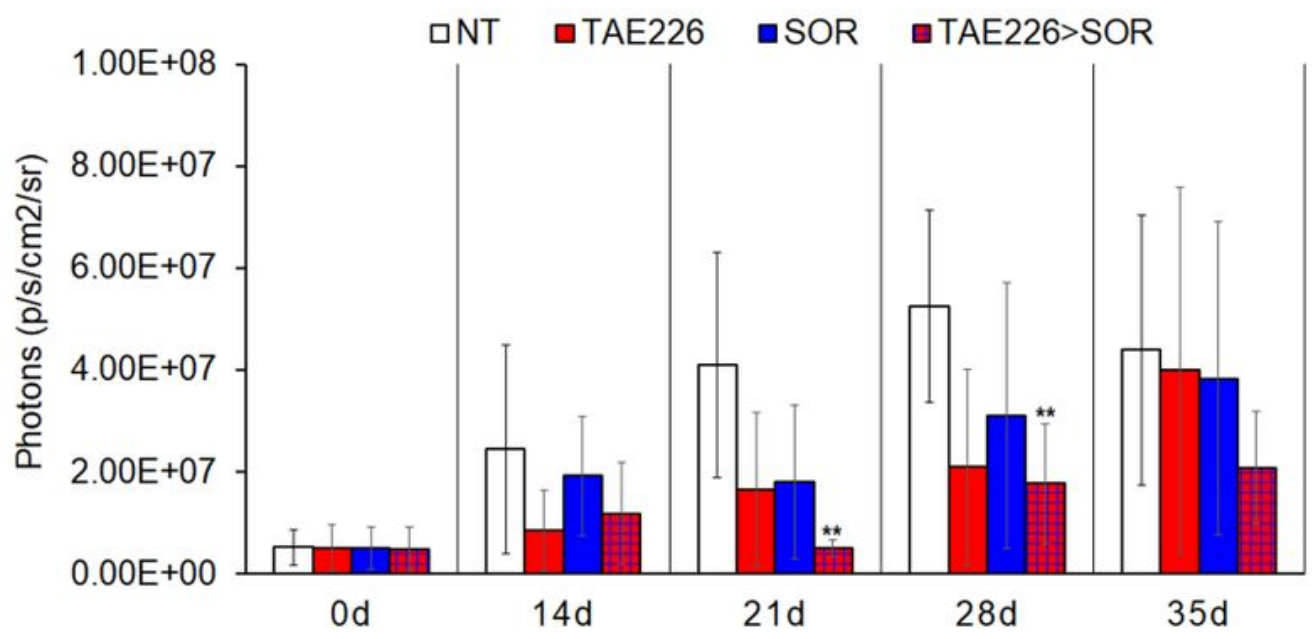

C

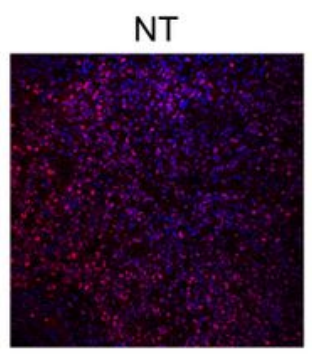

TAE226
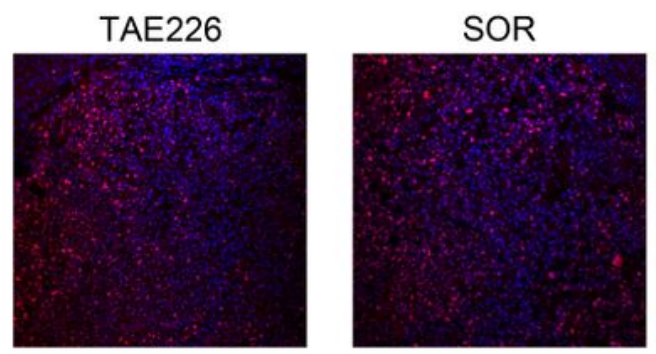

TAE226>SOR

D
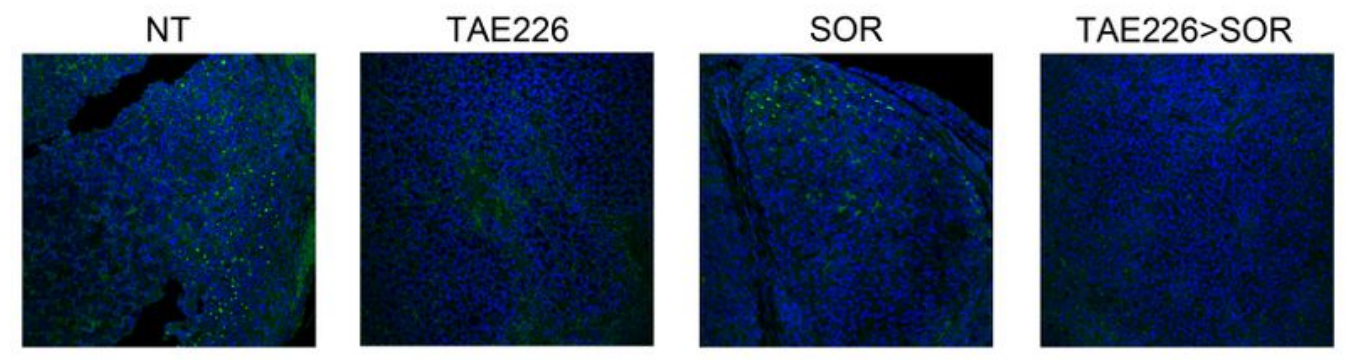

\section{Figure 4}

Effect of TAE226>SOR on HCC growth in mouse xenograft model. (A) Scheme of the experimental design. Imaging analysis was performed at different times (I). (B) Quantitative analysis of luciferase activity in vivo at various time points. The luminescence signals are expressed as mean $\pm S D$ of total flux of photon/sec/cm2/steradian (p/s/cm2/sr). Data were analyzed by ANOVA test. $(\star \star p<0.01 ; n=6)$. Representative images of immunofluorescence for PCNA (in red) (C) and pTyr397FAK (in green) (D) in 
mouse xenograft models after treatments. The nuclei are revealed by specific DAPI staining, displayed in blue. 40X Magnification.

\section{A}

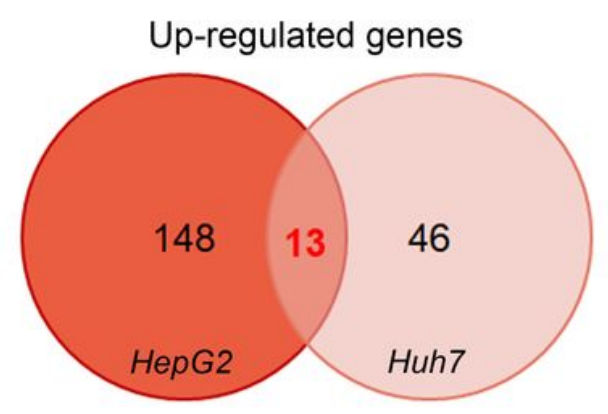

Down-regulated genes

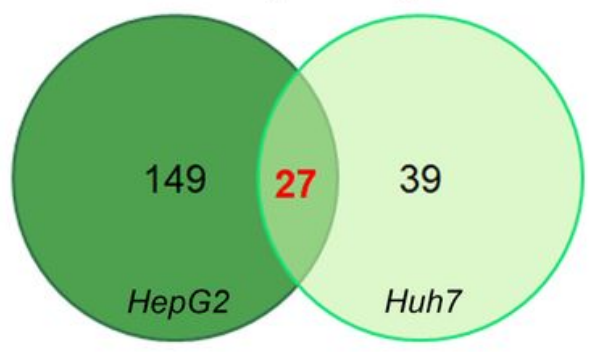

B
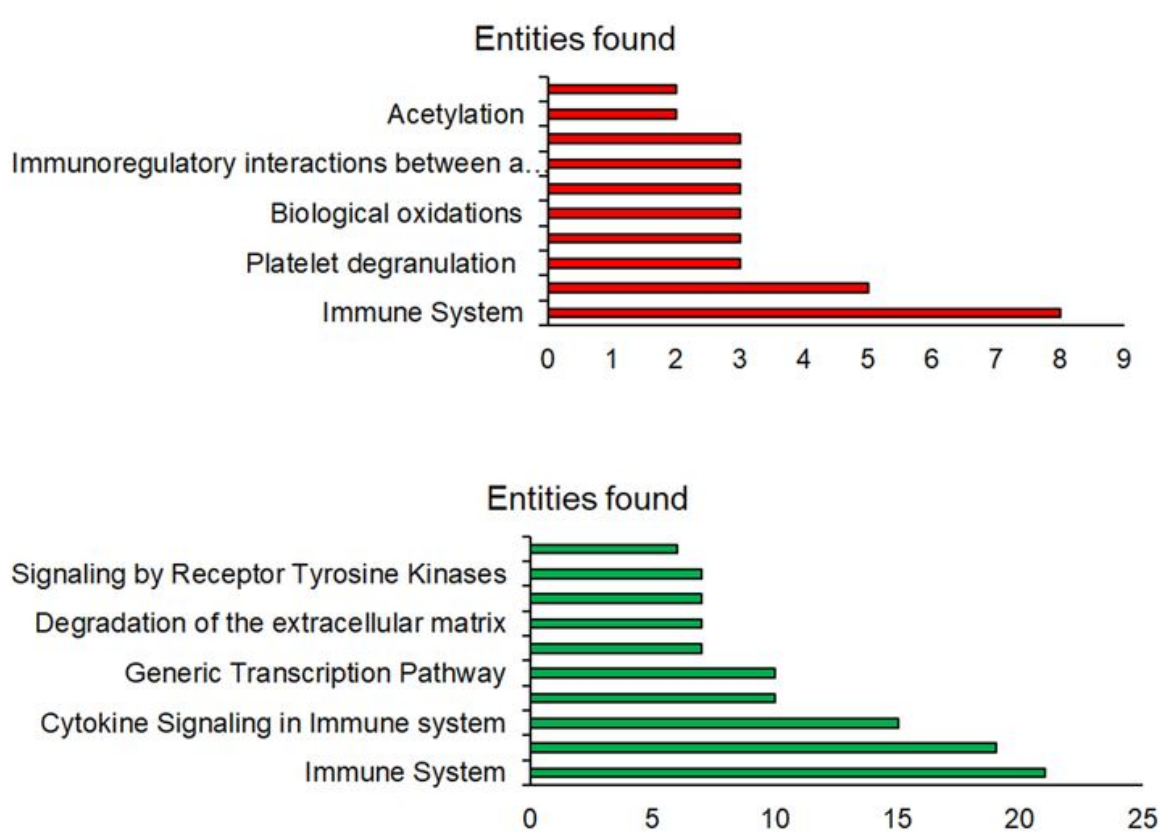

Figure 5

Cancer associated genes upon FAK inhibition. (A) Venn diagram showing the overlapping of up-regulated (upper circles) and down-regulated (lower circles) genes in HepG2 and HuH7 cells treated with TAE226>SOR compared to NT cells. (B) Bar plots of the 10 most abundant pathways for commonly upregulated (upper plot) or down-regulated (lower plot) genes in both HCC cells after treatment with TAE226>SOR. 
A

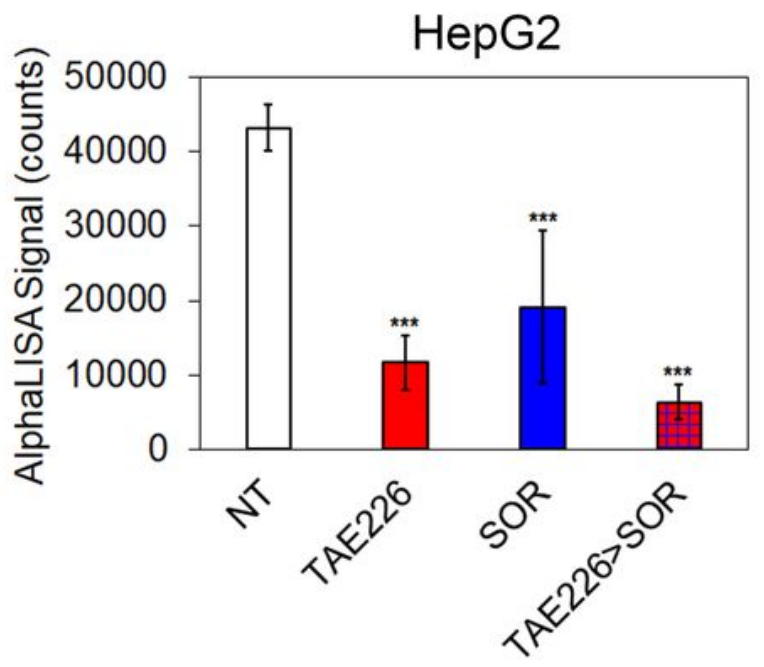

B
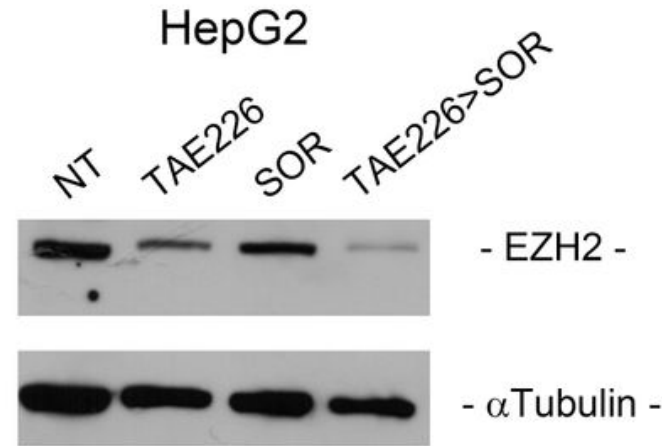

C
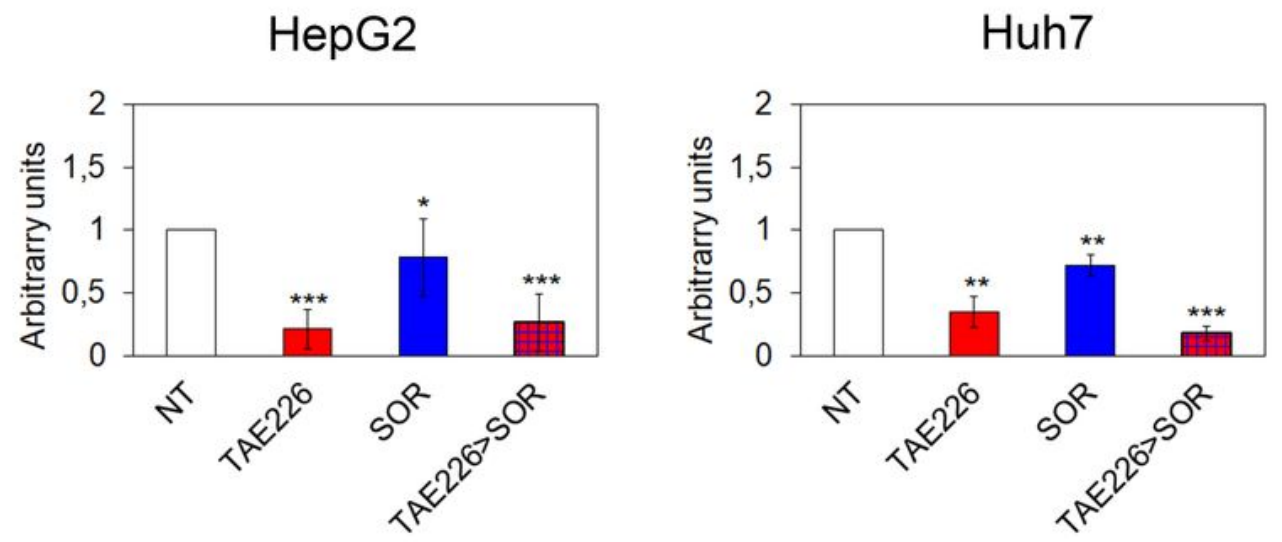

\section{Figure 6}

Effect of TAE226>SOR on H3K27me3. (A) H3K27me3 levels measured by AlphaLISA assay. Representative immunoblot (B) and quantitative analysis (C) of EZH2 expression after treatments, in HepG2 and Huh7 cells. Values are the mean of arbitrary units \pm SD of at least three independent experiments. Data were analyzed by 2 -tailed Student's t test. ${ }^{\star} p<0.05$; ${ }^{\star \star} p<0.01$; ${ }^{\star \star \star} p<0.001$ vs. NT. 
A
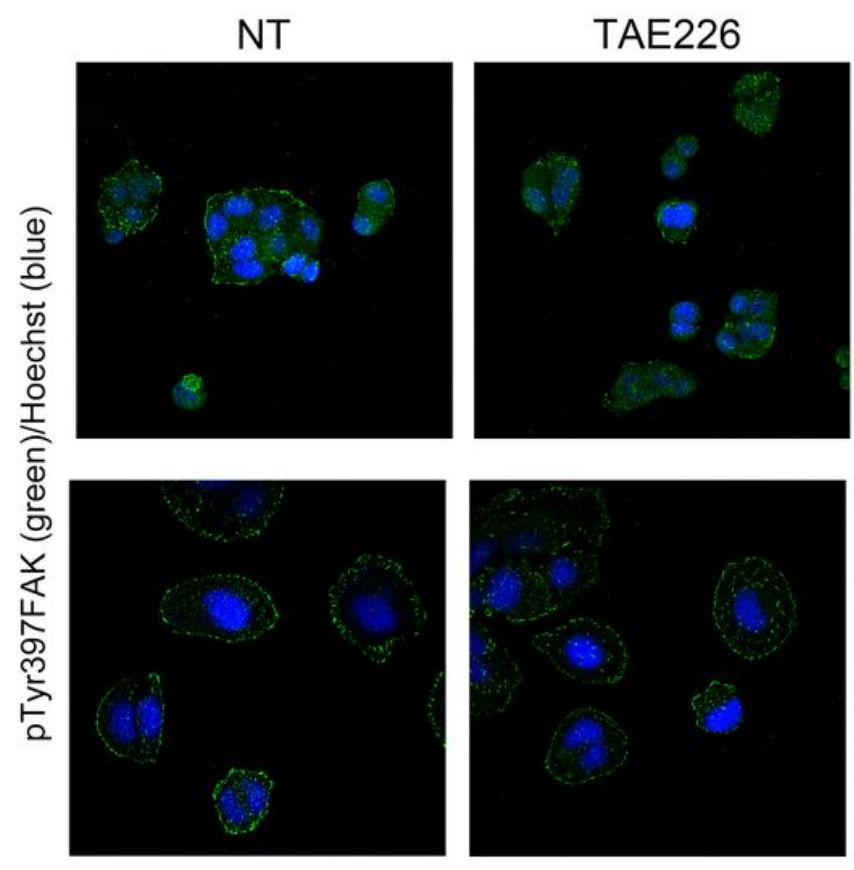

B
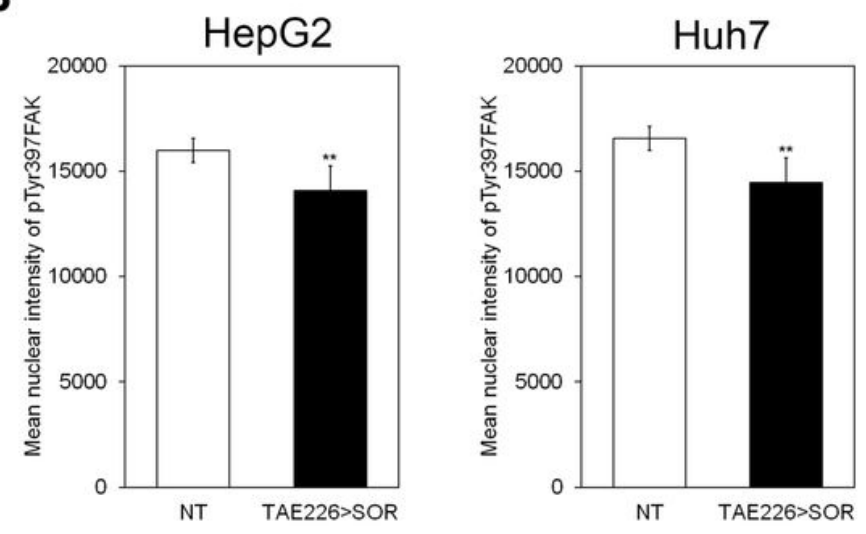

D NT

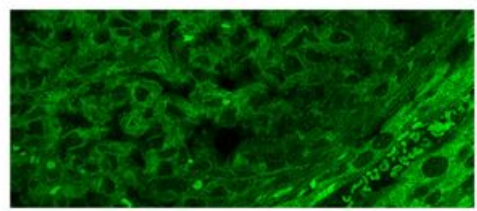

TAE226

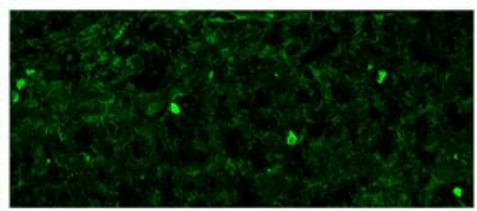

SOR
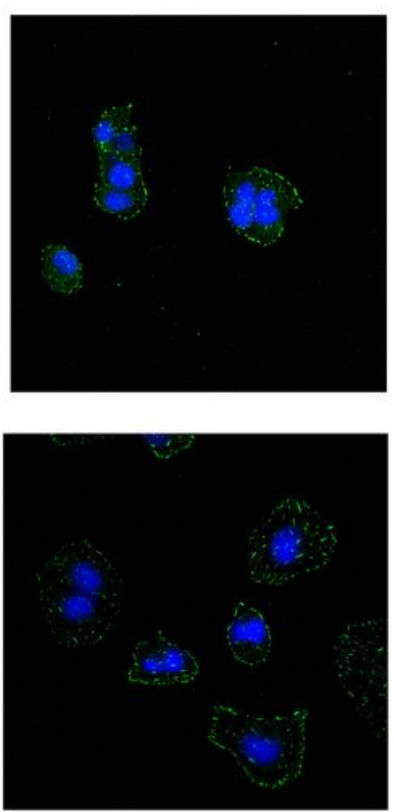

C
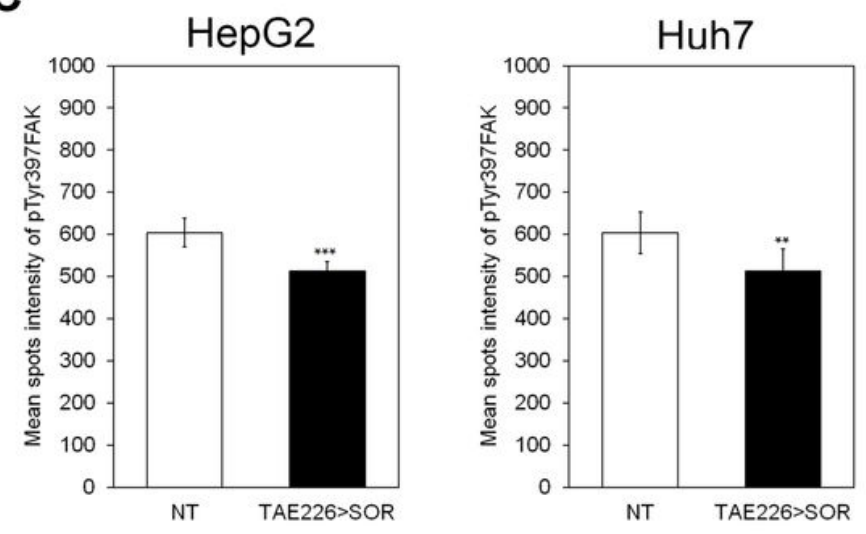

SOR

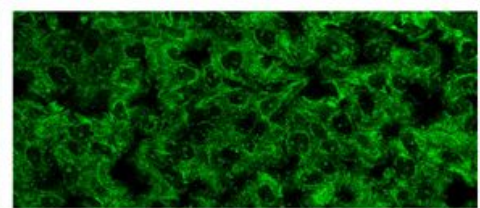

TAE226>SOR
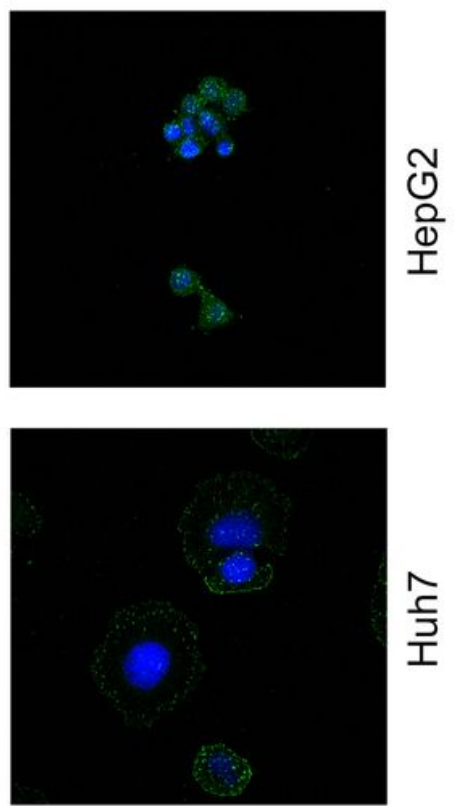

속

\section{Figure 7}

Effect of TAE226>SOR on nuclear amount of pTyr397FAK. (A) Representative images of immunofluorescence for pTyr397FAK (in green) into the nuclei and at focal adhesions after treatments, in HepG2 and Huh7 cells. The nuclei are revealed by specific Hoechst staining (in blue). 40X Magnification. Content of pTyr397FAK into the nuclei (B) and at focal adhesions (C) in HepG2 and Huh7 cells, quantified by using the Operetta CLS in confocal mode. 40X Magnification. (D) Representative images of immunofluorescence for pTyr397FAK (in green) in transgenic models of HCC. 60X Magnification. Values 
are the mean of arbitrary units \pm SD of at least three independent experiments. Data were analyzed by 2tailed Student's t test. ${ }^{*} p<0.05 ;{ }^{* *} p<0.01 ; * * * p<0.001$ vs. NT.

A

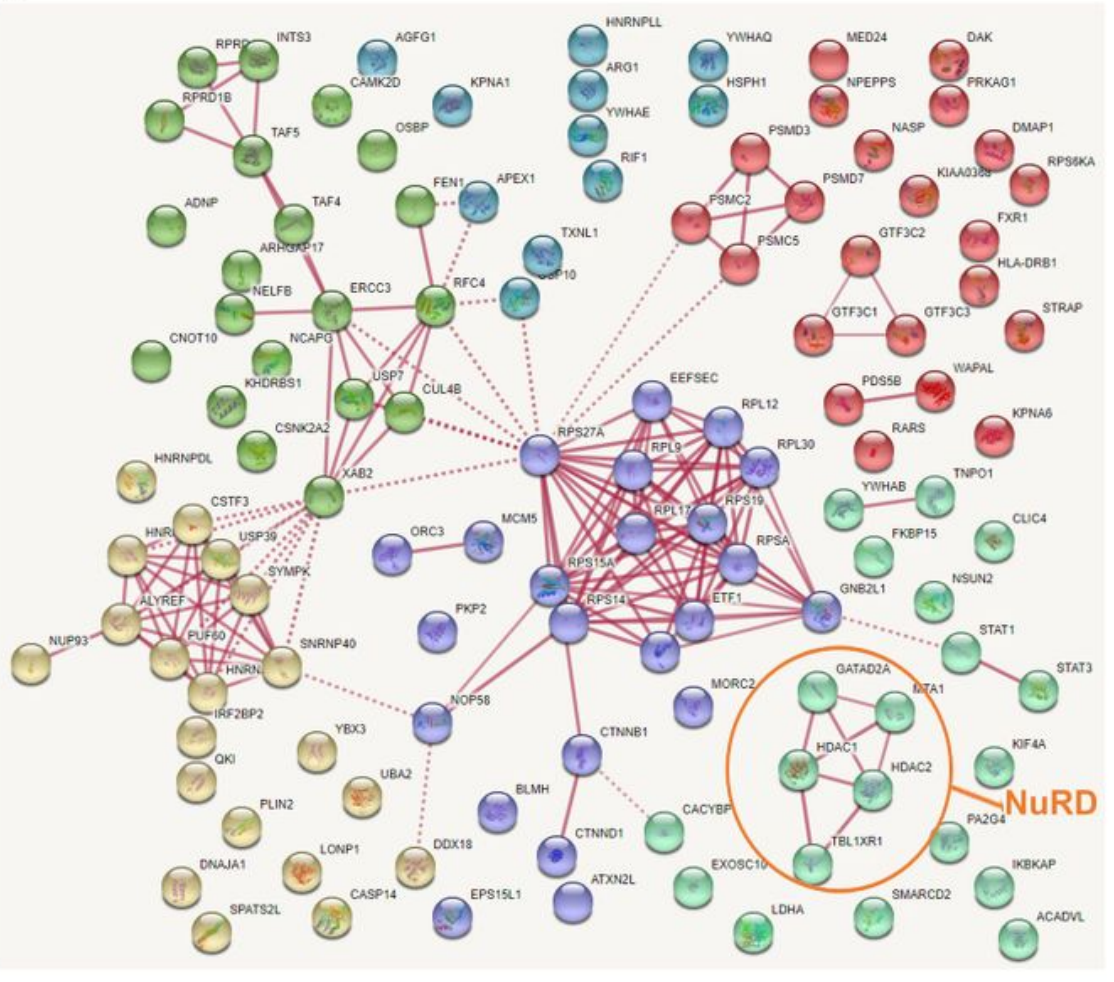

D
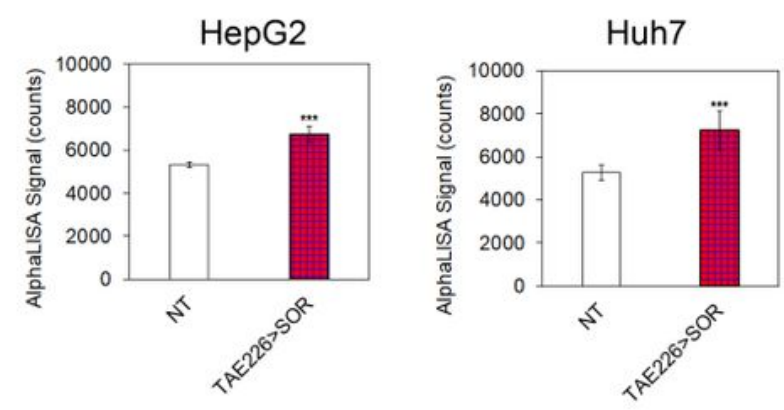

E
HCC cell

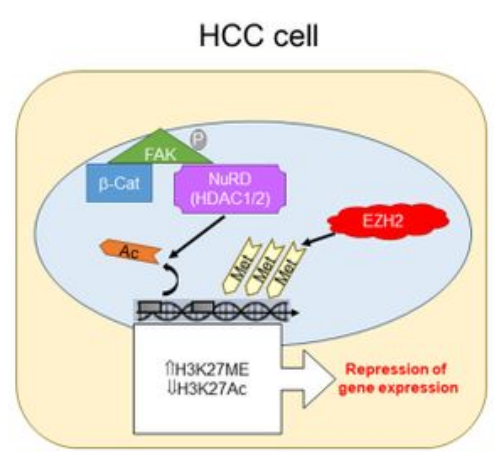

\section{E}

B

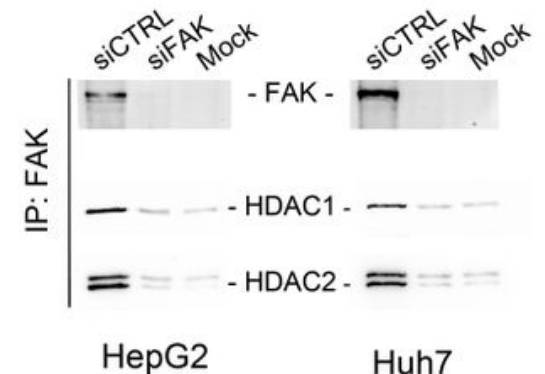

C
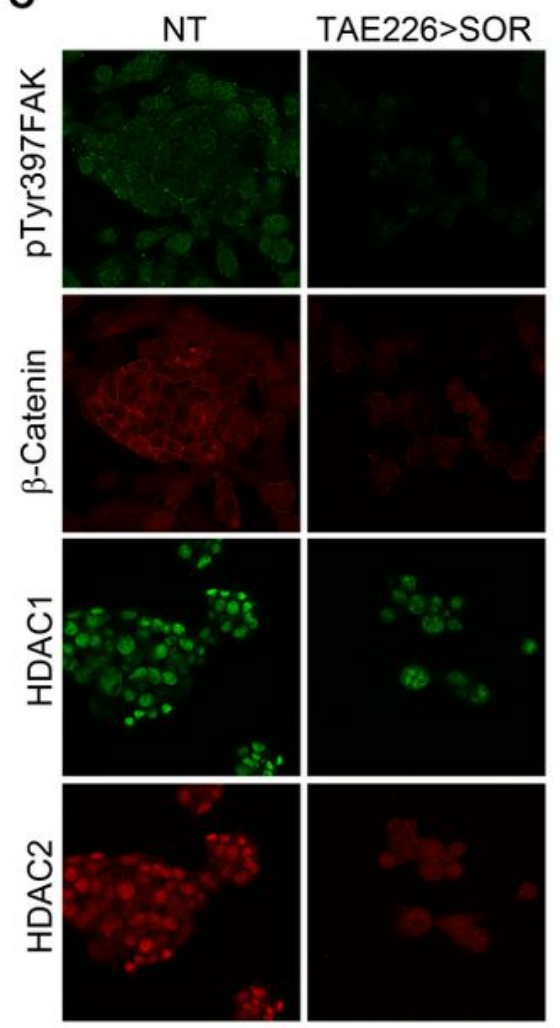

HCC cell treated with TAE226>SOR

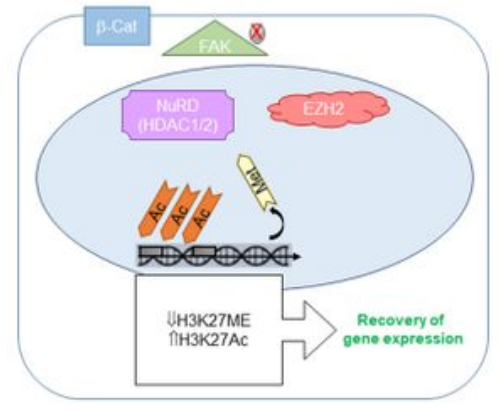

Figure 8

Effects of FAK inhibition on its nuclear interactome. (A) PPI networks of the nuclear FAK interactors. (B) Representative immunoblot of nuclear extracts immunoprecipitated (IP) with antibody against FAK and then immunoblotted with FAK, HDAC1, and HDAC2 antibodies in HCC cells silenced or not for FAK and in 
MOCK. (C) Representative images of immunofluorescence for pTyr397FAK, \-Catenin, HDAC1 and HDAC2 in HepG2 cells NT and after TAE226>SOR treatment. 60X Magnification. (D) H3K27ac levels measured by AlphaLISA assay. (E) Graphical abstract reporting the interaction of FAK with NuRD complex.

\section{Supplementary Files}

This is a list of supplementary files associated with this preprint. Click to download.

- SupplementaryMaterial09092021JECCRtobesubmitted.docx

- TableS4Nuclearlnteractome.xlsx 